\title{
Security Analysis of SKINNY under Related-Tweakey Settings
}

\author{
Guozhen Liu ${ }^{2,1}$, Mohona Ghosh ${ }^{1,3}$ and Ling Song ${ }^{1,4(\varangle)}$ \\ ${ }^{1}$ Nanyang Technological University, Singapore, Singapore \\ ${ }^{2}$ School of Cyber Science and Engineering, Shanghai Jiao Tong University, Shanghai, China \\ liuguozhen@sjtu.edu.cn \\ ${ }^{3}$ Indian Institute of Information Technology, Design and Manufacturing (IIITDM), Jabalpur, \\ India \\ mohona@iitdmj.ac.in \\ ${ }^{4}$ State Key Laboratory of Information Security (SKLOIS), Institute of Information Engineering, \\ Chinese Academy of Sciences, Beijing, China \\ songling@iie.ac.cn
}

\begin{abstract}
.
In CRYPTO'16, a new family of tweakable lightweight block ciphers - SKINNY was introduced. Denoting the variants of SKINNY as SKINNY- $n-t$, where $n$ represents the block size and $t$ represents the tweakey length, the design specifies $t \in\{n, 2 n, 3 n\}$. In this work, we evaluate the security of SKINNY against differential cryptanalysis in the related-tweakey model. First, we investigate truncated related-tweakey differential trails of SKINNY and search for the longest impossible and rectangle distinguishers where there is only one active cell in the input and the output. Based on the distinguishers obtained, 19, 23 and 27 rounds of SKINNY- $n-n$, SKINNY- $n-2 n$ and SKINNY- $n-3 n$ can be attacked respectively. Next, actual differential trails for SKINNY under related-tweakey model are explored and optimal differential trails of SKINNY-64 within certain number of rounds are searched with an indirect searching method based on Mixed-Integer Linear Programming. The results show a trend that as the number of rounds increases, the probability of optimal differential trails is much lower than the probability derived from the lower bounds of active Sboxes in SKINNY.
\end{abstract}

Keywords: Lightweight Block Cipher - SKINNY - Impossible Differential Attack • Rectangle Attack · Related-Tweakey

\section{Introduction}

Ubiquitous computing is rapidly emerging as the new computing paradigm in information technology sector. The mass development and deployment of pervasive devices such as RFID tags, sensors, smartcards etc. promise many benefits such as lower implementation costs, optimized performance and increased efficiency. At the same time, these devices demand harsh costs constraints like lower memory availability, lower area requirements and power constraints. Ensuring strong security from cryptographic point of view under such circumstances becomes a striving issue. Lightweight cryptography is a field of cryptography which encompasses the current state-of-the-art cryptographic algorithms that are tailored for implementation in constrained environments and directly cater to the security concerns of low cost devices. With the growing interest of the symmetric cryptographic community in this field, several lightweight variants of traditional cryptographic primitives such as 
lightweight block ciphers (PRESENT [BKL $\left.{ }^{+} 07\right]$, LED [GPPR11], SIMON [BSS $\left.{ }^{+} 13\right]$ etc.), lightweight hash functions (Spongent [BKL $\left.{ }^{+} 11\right]$, Photon [GPP11], Quark [AHMN13] etc.) and lightweight stream ciphers (Mickey [BD08], Grain [HJMM08], Trivium [CP08] etc.) have been proposed and studied in literature to address the design and security goals of lightweight ciphers.

In this work, we focus on the security analysis of SKINNY family of lightweight block ciphers. Proposed by Beierle et al. in CRYPTO'16 [BJK $\left.{ }^{+} 16 \mathrm{~b}\right]$, the design of SKINNY has many attractive features. Firstly, its design can be seen as a first step towards bridging the gap between high operational efficiency vs. strong security. By careful analysis and thorough investigation, the designers of SKINNY show how non-optimal but very light internal crypto components can be combined together to provide a cipher which has competitive performance as well as strong security guarantees in both single key as well as related key settings. Secondly, inspired by the TWEAKEY framework [JNP14], SKINNY replaces its key input with a tweakey input. This provides the users of SKINNY an added advantage of enjoying the benefits of a tweakable block cipher.

The official SKINNY specification $\left[\mathrm{BJK}^{+} 16 \mathrm{c}\right.$ ] defines two block sizes, i.e., 64-bit and 128-bit. Depending on the block length $n$, the tweakey length $t$ can be $n, 2 n$ or $3 n$. Consequently, if we denote a variant of SKINNY as SKINNY- $n-t$, then the six variants of SKINNY are - SKINNY-64-64, SKINNY-64-128, SKINNY-64-192, SKINNY-128-128, SKINNY-128-256 and SKINNY-128-384. In the design document of SKINNY [BJK ${ }^{+} 16 \mathrm{c}$ ], the designers provide a detailed security evaluation of SKINNY against the traditional block cipher cryptanalysis. However, unlike the differential and linear attacks, for which the lower bounds on the number of active S-boxes under the single key as well as the related-tweakey settings have been provided, for other attack types such as MITM, impossible differential, integral attacks etc., the analysis has been restricted to single key model only. Moreover, in these attacks, only SKINNY- $n-n$ variants have been investigated. This motivates us to analyze the security of all SKINNY variants under the related-tweakey model. We utilize related-tweakey impossible differential and rectangle attacks for our analysis.

Related Work. In September'16, the designers of SKINNY announced a competition where they invited the cryptographic community to cryptanalyse SKINNY-64-128 and SKINNY-128-128 under the following categories $\left[\mathrm{BJK}^{+} 16 \mathrm{a}\right]$.

- SKINNY-64-128: 18 - or 20- or 22- or 24- or 26-rounds

- SKINNY-128-128: 22- or 24- or 26- or 28- or 30-rounds

Following the announcement, several independent cryptanalytic results on SKINNY (including ours) were reported within a short time span of each other. Under the single tweakey settings, Tolba et al. [TAY16] demonstrated impossible differential attacks against 18,20 and 22 rounds of SKINNY- $n-n$, SKINNY- $n-2 n$ and SKINNY- $n-3 n$ respectively. In [SMB16], Sadeghi et al. investigated the security of SKINNY under zero-correlation linear cryptanalysis and constructed 9 and 10-round distinguishers for the same. They also reported 12-round impossible differential distinguishers for SKINNY-64 and SKINNY-128 under the related tweakey model. In [ABC $\left.{ }^{+} 17\right]$, Ankele et al. analyzed SKINNY-64-128 and presented 21, 22 and 23-round related tweakey impossible differential attacks which have a low time complexity but suffer from two limitations. Firstly, all their attacks have data complexity beyond codebook. Secondly, the 22 and 23-round attacks shown require certain tweakey bits to be public and at some specific cell positions. However, the position of the public tweakey cells chosen in their attacks do not conform with that recommended in the original SKINNY design document $\left[\mathrm{BJK}^{+} 16 \mathrm{c}\right]$, thus their attacks were shown on a modified version of SKINNY-64/128. 
Our Contribution. Our main results are summarized in Table 1. We mainly focus on attacks which have a data complexity below codebook and for which the whole tweakey input is secret. For most of our attacks, we employ truncated differential trails to serve our purpose. In all these results, we construct distinguishers where there is only one active cell in the input and the output difference. Moreover, the position of the active cell has been chosen such that maximum number of rounds can be extended in the forward and backward direction from the distinguisher. Under these conditions, the distinguishers so constructed are the longest. Based on the truncated distinguishers, 19, 23 and 27 rounds of SKINNY- $n-n$, SKINNY- $n-2 n$ and SKINNY- $n-3 n$ can be attacked respectively. Additionally, extensions to the case where a tweak is used are discussed for SKINNY- $n$ - $2 n$ and SKINNY- $n-3 n$ under impossible attacks.

We also analyze actual differential trails for SKINNY under related-tweakey model. Through our rectangle attacks based on the actual trails, it is shown that employing actual differential trails help in getting longer distinguishers and sometimes better cryptanalytic results. What's more, optimal trails of SKINNY-64 within certain number of rounds show a positive trend for the designers that as the number of rounds increases, the probability of optimal differential trails is much lower than the probability derived from the lower bounds of active Sboxes in SKINNY.

Table 1: Summary of cryptanalytic results on SKINNY

\begin{tabular}{|c|c|c|c|c|c|c|c|c|}
\hline Vers. & $n$ & Rounds & $\operatorname{Data}^{\dagger}$ & Time & Memory & Dist. Type & Attack & Ref. \\
\hline \multirow{2}{*}{$n-n$} & 64 & 19 & $2^{61.47}$ & $2^{63.03}$ & $2^{56}$ & Trunc. Diff. & Imposs. & \multirow{2}{*}{$\S 3$} \\
\hline & 128 & 19 & $2^{122.47}$ & $2^{124.60}$ & $2^{112}$ & Trunc. Diff. & Imposs. & \\
\hline \multirow{2}{*}{$n-2 n$} & 64 & 23 & $2^{62.47}$ & $2^{125.91}$ & $2^{124}$ & Trunc. Diff. & Imposs. & \multirow{2}{*}{$\S 3$} \\
\hline & 128 & 23 & $2^{124.47}$ & $2^{251.47}$ & $2^{248}$ & Trunc. Diff. & Imposs. & \\
\hline \multirow{3}{*}{$n-3 n$} & 64 & 27 & $2^{63.5}$ & $2^{165.5}$ & $2^{80}$ & Trunc. Diff. & Rect. & \multirow{2}{*}{$\S 4$} \\
\hline & 128 & 27 & $2^{127}$ & $2^{351}$ & $2^{160}$ & Trunc. Diff. & Rect. & \\
\hline & 128 & 27 & $2^{112}$ & $2^{331}$ & $2^{144}$ & Diff. & Rect. & $\S 5$ \\
\hline
\end{tabular}

$\dagger$ Attacks with a data complexity below codebook are focused.

Organization. This work is organized as follows. In Section 2, we provide a brief description of SKINNY and its properties, followed by the important notations adopted throughout the work. Section 3 gives details of the 23-round attack on SKINNY-n$2 n$ and results on other SKINNY variants under related-tweakey impossible differential attack. Section 4 discusses the 22-round attack on SKINNY- $n$ - $2 n$ using the related-tweakey rectangle attack and presents results on other variants. In the above sections, truncated differential trails are used for analysis. In Section 5, we investigate actual differential characteristics for SKINNY and present related-tweakey rectangle attacks based on them. Section 6 summarizes and concludes this paper.

\section{Preliminaries}

In this section, we first describe SKINNY and then mention the key notations and definitions used in our cryptanalysis to facilitate better understanding. 


\subsection{Description of SKINNY}

The SKINNY block cipher adopts substitution-permutation network and elements of TWEAKEY framework [JNP14] in its design. Represented as SKINNY- $n$ - $t$, where, $n / t$ denotes the block size/ tweakey size respectively, this block cipher has six variants namely - SKINNY-64-64, SKINNY-64-128, SKINNY-64-192, SKINNY-128-128, SKINNY-128-256 and SKINNY-128-384. The number of rounds in each variant are 32, 36, 40, 40, 48 and 56 respectively. Both the 64-bit and 128-bit internal states are represented as $4 \times 4$ array of cells with each cell being a nibble in case of $n=64$ bits and a byte in case of $n=128$ bits. The tweakey state is seen as a group of $z 4 \times 4$ arrays, where, $z=\frac{t}{n}$ and $z \in\{1,2,3\}$. The arrays are marked as $T K-1 / T K-1, T K-2 / T K-1, T K-2, T K-3$ for $z=1 / 2 / 3$ respectively. In all the cases, the cells are numbered row-wise as shown in Fig. 1. Each round consists of 5 basic operations as shown in Fig. 2.

\begin{tabular}{|c|c|c|c|}
\hline 0 & 1 & 2 & 3 \\
\hline 4 & 5 & 6 & 7 \\
\hline 8 & 9 & 10 & 11 \\
\hline 12 & 13 & 14 & 15 \\
\hline
\end{tabular}

Figure 1: Cell numbering in a state of SKINNY

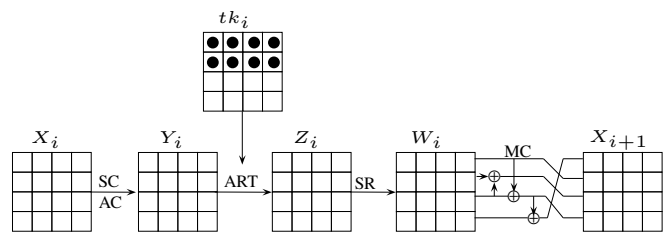

Figure 2: $i^{\text {th }}$ round of SKINNY. Only the first two rows (cells marked with $\bullet$ ) in the round tweakey $t k_{i}$ are xor'ed in each round

1. SubCells $(S C)$ - The non-linear substitution layer uses a 4-bit S-box in case of $n=64$ bits and a 8 -bit S-box in case of $n=128$ bits. The maximal differential probability of both 4 -bit and 8 -bit S-boxes is $2^{-2}$.

2. AddConstants $(A C)$ - This step involves xoring three round constants to the first three cells of the first column of an internal state.

3. AddRoundTweakey (ART) - In this step, the first two rows of the round tweakey $\left(t k_{i}\right)$ are xor'ed with the first two rows of the corresponding internal state. The round tweakey $\left(t k_{i}\right)$ is defined as:

- $z=1: t k_{i}=(T K-1)_{i}$

- $z=2: t k_{i}=(T K-1)_{i} \oplus(T K-2)_{i}$

- $z=3: t k_{i}=(T K-1)_{i} \oplus(T K-2)_{i} \oplus(T K-3)_{i}$

The tweakeys $(T K-1)_{i},(T K-2)_{i}$ and $(T K-3)_{i}$ for each round $i$ are generated by a tweakey scheduling algorithm.

4. ShiftRows (SR) - The linear shift rows operation performs circular right shift on each row of the internal state. The number of shifts in each row $j$ is $j$ for $0 \leq j \leq 3$.

5. MixColumns $(M C)$ - This linear transformation pre-multiplies each column of the internal state by a $4 \times 4$ binary matrix $M$ shown below. The inverse MixColumns operation $\left(M^{-1}\right)$ can be computed as shown below.

$$
M=\left(\begin{array}{cccc}
1 & 0 & 1 & 1 \\
1 & 0 & 0 & 0 \\
0 & 1 & 1 & 0 \\
1 & 0 & 1 & 0
\end{array}\right) \quad M^{-1}=\left(\begin{array}{cccc}
0 & 1 & 0 & 0 \\
0 & 1 & 1 & 1 \\
0 & 1 & 0 & 1 \\
1 & 0 & 0 & 1
\end{array}\right)
$$


Tweakey Scheduling Algorithm (TSA). - The tweakey schedule of SKINNY is a linear scheduling algorithm. The tweakey input is first loaded with a $n, 2 n$ or $3 n$-bit tweakey input. Accordingly, we have $T K-1$ with $z=1$ or $(T K-1, T K-2)$ with $z=2$ or $(T K-1$, $T K-2, T K-3$ ) with $z=3$. The cells in each of these $4 \times 4 T K-m$ arrays (for $m \in\{1,2,3\}$ ) are numbered row-wise. The round tweakeys are then generated as follows:

- Permutation Phase: In this phase, a permutation $P$ defined as:

$$
P=[9,15,8,13,10,14,12,11,0,1,2,3,4,5,6,7]
$$

is first applied to each of the $T K-m$ arrays as: $T K-m[i] \leftarrow T K-m[P[i]]$ for all $0 \leq i \leq 15$ depending on $z=1,2$ or 3 .

- LFSR Update Phase: In this phase, all cells of the first two rows of $T K-2$ / (TK-2, $T K-3)$ for $z=2 / 3$ respectively are individually updated using a 4-bit (if the cell is a nibble) or a 8-bit (if the cell is a byte) LFSR. Note that $T K-1$ is not updated in this phase.

The first two rows of each of the $(T K-m)_{i}$ arrays are used to generate the corresponding round tweakey $t k_{i}$ as discussed earlier. This process is repeated until all round tweakeys have been generated.

Case when tweakey state comprises of both the key and the tweak bits. The SKINNY specification allows the tweakey state to be filled with both tweak (public) and key (secret) material with the condition that the key size should always be at least as big as the block size. Further, the tweak material is loaded into $T K-1$ as recommended by the designers. This work follows these recommendations in attacks where a tweak is used.

For complete details of the state updation process and the tweakey scheduling algorithm, one can refer $\left[\mathrm{BJK}^{+} 16 \mathrm{~b}\right]$.

\subsection{Notations and Definitions}

The following notations are followed throughout the rest of the paper.

\begin{tabular}{|c|c|c|}
\hline c & : & Cell size, where $c \in\{4,8\}$ \\
\hline $\mathbf{n}$ & $:$ & Block size, where $n \in\{64,128\}$ \\
\hline $\mathbf{R}_{\mathrm{d}}$ & $:$ & Rounds covered by the distingusiher \\
\hline $\mathbf{R}_{\mathrm{b}}$ & : & Rounds extended backward in the tweakey recovery phase \\
\hline $\mathbf{R}_{\mathbf{f}}$ & $:$ & Rounds extended forward in the tweakey recovery phase \\
\hline $\mathrm{tk}_{\mathrm{i}}$ & : & Round tweakey of round $i$ \\
\hline $\mathbf{X}_{\mathbf{i}}$ & $:$ & State before $\mathrm{SC}, \mathrm{AC}$ in round $i$ \\
\hline $\mathbf{Y}_{\mathbf{i}}$ & : & State before ART in round $i$ \\
\hline $\mathbf{Z}_{\mathbf{i}}$ & : & State before SR in round $i$ \\
\hline $\mathbf{W}_{\mathbf{i}}$ & : & State before MC in round $i$ \\
\hline $\operatorname{Row}(\mathbf{j})$ & : & $j^{\text {th }}$ row, where, $1 \leq j \leq 4$ \\
\hline $\operatorname{Col}(\mathrm{k})$ & : & $k^{t h}$ column, where, $1 \leq k \leq 4$ \\
\hline $\mathbf{S}$ & : & Substitution of Sbox \\
\hline$\Delta \mathrm{X}$ & : & Difference in a state $X$ \\
\hline $\mathbf{X}_{\mathbf{i}}[\mathbf{m}]$ & $:$ & $m^{t h}$ cell of a state $X$ in round $i$, where $0 \leq m \leq 15$ \\
\hline $\mathbf{X}_{\mathbf{i}}[\mathbf{p}, \ldots$, & : & $p^{t h}$ cell, $\ldots, r^{t h}$ cell of state $X$ in round $i$, where $0 \leq p, r$ \\
\hline
\end{tabular}

The time complexity of the attack is measured in terms of number of $r$-round SKINNY encryptions required and the memory accesses. The memory complexity is measured in units of 64-/128-bit SKINNY blocks required. 
In $\left[\mathrm{BJK}^{+} 16 \mathrm{~b}\right]$, the designers of SKINNY define the adversarial model to be $T K 1$, $T K 2$ and $T K 3$ for the scenarios where an attacker can inject differences in the tweakey state based on the respective SKINNY variants used. We follow the same notation in our attacks.

\subsection{Properties of SKINNY}

Some interesting properties of SKINNY that were utilized during our attacks are as follows:

1. The SKINNY MixColumns matrix is not an MDS matrix. Therefore, during the tweakey recovery phase of an attack, sometimes it is not enough to know the values of only the active cells in the output column of $M C$ operation to determine the value of the active cell in the input column, and vice versa. This leads to more cells need to be guessed. To highlight this issue, consider Fig. 3.

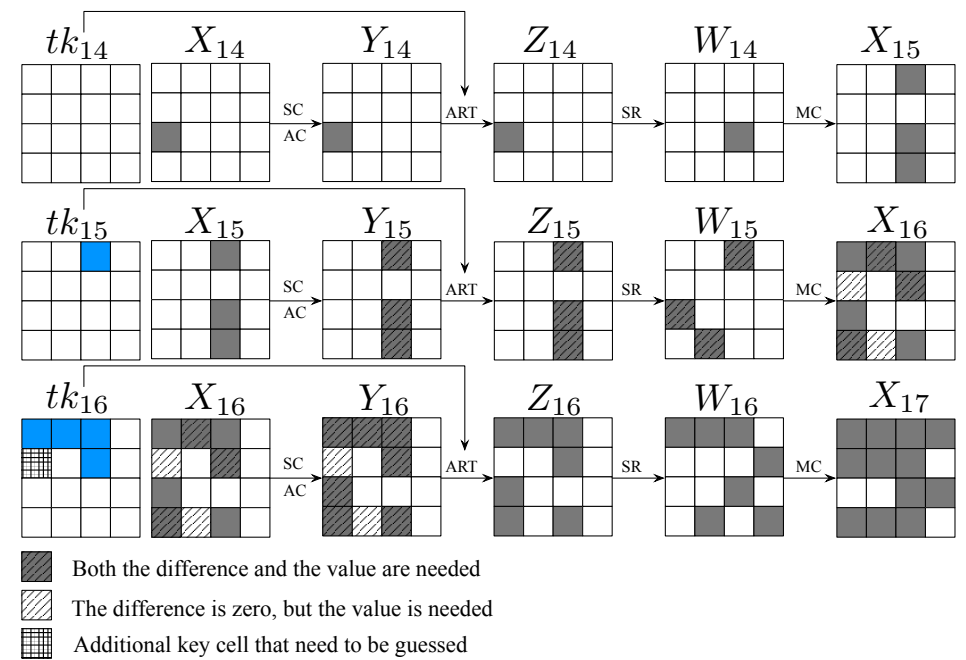

Figure 3: Property of MixColumns of SKINNY

In this figure, we have a truncated differential trail with probability 1 from $\Delta X_{14}$ to $\Delta X_{17}$. Now, if we backtrack this trail from $\Delta X_{17}$, then to guarantee that there would be only one-cell difference in $\Delta X_{14}[8]$, it is necessary and sufficient to check whether the 3 cell difference in $\Delta X_{15}[2,10,14]$ leads to one cell difference in $\Delta W_{14}[10]$ or not. To this, knowledge of $\Delta X_{15}[2,10,14]$ is required, and thus the differences as well as actual state values at $Y_{15}[2,10,14]\left(=W_{15}[2,8,13]\right)$ are required. Now, if suppose, values as well as differences in the active cells of the first column of $X_{16}$ are known, only $\Delta W_{15}[8]$ can be computed. To compute $W_{15}[8]$, the value of $X_{16}$ [4] needs to be known as well since $W_{15}[8]=X_{16}[4] \oplus X_{16}[12]$. This in turn leads to an additional tweakey cell guess in $t k_{16}$ as $t k_{16}$ [4] needs to be guessed to determine $X_{16}$ [4].

This requirement of guessing more cells serves as one of the factors in determining the number of rounds covered in the tweakey recovery phase of our attacks.

2. The order of $A R T, S R$ and $M C$ operations in any round can be changed by first applying $S R$ and $M C$ operations and then xoring the intermediate state with an equivalent round tweakey input. We denote this equivalent tweakey by $t k^{e q}=$ $M C(S R(t k))$ as shown in Fig. 4. 


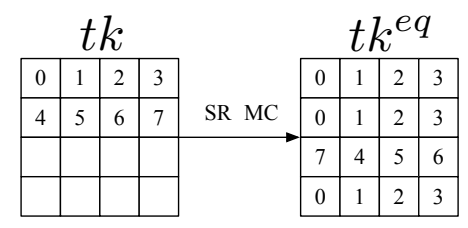

Figure 4: Reordering of $A R T, S R$ and $M C$ operations in a round

3. The TSA of SKINNY is linear. Hence, if we know the value of differences injected in the master tweakey, the exact differences in all the other round keys can be determined.

4. Only the first two rows of each round tweakey are xor'ed with the intermediate state in each round. Thus, the tweakey cells involved in the $i^{\text {th }}$ round will next appear in $(i+2)^{t h}$ round and so on. Moreover, in the tweakey schedule algorithm, the same cell-wise permutation is applied synchronously (at the same time) to each of the tweakey arrays and then each of the tweakey cells of the first two rows of $T K-2$ and $T K-3$ are updated with LFSRs leading to a diffusion that only happens within cells.

5. Subtweakey difference cancellation. As noticed by the designers $\left[\mathrm{BJK}{ }^{+} 16 \mathrm{c}\right]$, for a given active tweakey cell, only a single subtweakey difference cancellation can happen every 30 rounds for TK2, and two subtweakey difference cancellations for TK3. Let us take TK2 as an example. Suppose that a single cell of $T K-1$ and $T K-2$ are active and the cell position is in the first two rows. Let $a_{1}$ and $a_{2}$ be differences of the active cells respectively. Then the subtweakey difference in the first round is $a_{1} \oplus a_{2}$ at this cell, and in the $(2 i+1)^{t h}$ round, the subtweakey difference is $a_{1}+\operatorname{LFSR} R_{2}^{i}\left(a_{2}\right)$ by ignoring the position permutation $P$. Since $a_{1}$ and $a_{2}$ are non-zero differences, and the $L F S R_{2}$ has a cycle of 15 , then a single subtweakey cancellation, i.e., $a_{1}+\operatorname{LFSR} R_{2}^{i}\left(a_{2}\right)=0$, can happen every 30 rounds. Under the case of TK3 where $a_{1}, a_{2}$ and $a_{3}$ are differences of the active cells, subtweakey difference cancellations, i.e., $a_{1}+\operatorname{LFS} R_{2}^{i}\left(a_{2}\right)+\operatorname{LFSR}_{3}^{i}\left(a_{3}\right)=0$ can happen at most twice every 30 rounds. By combining Property 4 with subtweakey difference cancellations, it is deduced that there can be three and five rounds of fully inactive internal states for TK2 and TK3 respectively.

We also utilize the following Lemma in our attacks.

Lemma 1. For any given $S K I N N Y S$-box $(c=4$ or 8) $S$ and any non-zero input - output difference pair $\left(\delta_{i n}, \delta_{\text {out }}\right)$, there exists one solution $y$ on average, for which the equation, $S_{i}(y) \oplus S_{i}\left(y \oplus \delta_{\text {in }}\right)=\delta_{\text {out }}$, holds true.

\section{Related-Tweakey Impossible Differential Attack}

In this section, we present our related-tweakey impossible differential attack on SKINNY. We investigate truncated impossible differential trails of SKINNY under certain fixed tweakey differences to mount our attacks. Impossible differential cryptanalysis was first proposed independently by Biham et al. [BBS05] and Knudsen [Knu98]. The main idea of this attack is to find an input difference that can never lead to a particular output difference, i.e., the probability of such a differential trail is zero. Then, one can derive the right key by discarding the keys which suggest this impossible differential. Under related-tweakey settings, the development of this differential is studied for two encryptions 
under related-tweakeys, where the relation between the two secret tweakeys is assumed to be known to the attacker.

Through our related-tweakey impossible differential attacks, 19, 23 and 27 rounds of SKINNY under $T K 1, T K 2$ and $T K 3$ are analyzed respectively. In this section, details of the 23-round attack on SKINNY- $n-2 n$ are discussed and extended to the cases where part of the tweakey is tweak. The attacks on other variants work in a similar manner and are presented in Appendix B. The attack consists of two phases: Distinguisher construction phase and Tweakey recovery phase.

\subsection{Related-Tweakey Impossible Distinguisher}

In this phase, we first construct a 14-round related-tweakey impossible distinguisher for SKINNY- $n$ - $2 n$ exploiting the subtweakey difference cancellation property (Property 5). Our distinguisher is placed between Round 5 to Round 19. A 7.5-round related-tweakey differential in the forward direction (having prob. 1) starting at $Y_{5}$ (after the $S C$ and $A C$ operations in Round 5) is concatenated to a 6.5-round related-tweakey differential (having prob. 1) starting in the reverse direction from $Y_{19}$ (before the $A R T$ operation in Round 19). The contradiction happens in Round 13 at $X_{13}[11]$. The 14-round related-tweakey impossible differential is:

$$
(00 b 0|0000| 0000 \mid 0000) \stackrel{14 y}{\longrightarrow}(0000|0000| 00 N 0 \mid 0000)
$$

where $b$ denotes a fixed non-zero difference and $N$ denotes any non-zero difference. The entire impossible differential path along with the round tweakey differences is illustrated in Fig. 5. The tweakey difference is only injected in one cell. The round tweakey differences used in the rounds involved in the key recovery phase are shown in Table 2. According to Property 5, for a given active cell, only a single subtweakey difference cancellation can happen every 30 rounds for TK2. In our case, we let the only cancellation take place at Round 7, i.e., $h=0 .{ }^{1}$

Table 2: The round tweakey differences $\Delta t k_{i}$ used in the key recovery phase.

\begin{tabular}{|c|c|c|}
\hline Round $i$ & Row(1) & Row $(2)$ \\
\hline 1 & $\left(\begin{array}{llll}0 & d & 0 & 0\end{array}\right)$ & $\left(\begin{array}{llll}0 & 0 & 0 & 0\end{array}\right)$ \\
\hline 3 & $\left(\begin{array}{llll}c & 0 & 0 & 0\end{array}\right)$ & $\left(\begin{array}{llll}0 & 0 & 0 & 0\end{array}\right)$ \\
\hline 5 & $\left(\begin{array}{llll}0 & 0 & b & 0\end{array}\right)$ & $\left(\begin{array}{llll}0 & 0 & 0 & 0\end{array}\right)$ \\
\hline 19 & $\left(\begin{array}{llll}a & 0 & 0 & 0\end{array}\right)$ & $\left(\begin{array}{llll}0 & 0 & 0 & 0\end{array}\right)$ \\
\hline 21 & $\left(\begin{array}{llll}0 & 0 & e & 0\end{array}\right)$ & $\left(\begin{array}{llll}0 & 0 & 0 & 0\end{array}\right)$ \\
\hline 23 & $\left(\begin{array}{llll}0 & 0 & 0 & 0\end{array}\right)$ & $\left(\begin{array}{llll}f & 0 & 0 & 0\end{array}\right)$ \\
\hline
\end{tabular}

We construct a 12-round distinguisher under TK1 and owing to the tweakey cancellations 16-round impossible distinguishers can be constructed under TK3. For more information regarding the impossible distinguishers under TK1 and TK3, we refer the readers to Appendix A.

\subsection{Tweakey Recovery Attacks}

In this section, we use the 14-round related-tweakey impossible differential distinguisher constructed earlier to attack 23-rounds of SKINNY- $n-2 n$ using a pair of related-tweakeys. Before the attack is explained, we introduce some more notations which are borrowed from [BNS14]. Suppose an impossible differential $(\Delta X \nrightarrow \Delta Y)$ has been constructed for

\footnotetext{
${ }^{1}$ As discussed earlier in Property 5, suppose the one-cell tweakey differences of $(T K-1)$ and $(T K-2)$ are $a_{1}$ and $a_{2}$ respectively. If we set $a_{1} \oplus a_{2}=d=5$ and $a_{1} \oplus \operatorname{LFSR} R_{2}^{6}\left(a_{2}\right)=h=0$, then differences $a, b, c, d, e, f, h, r, s, t, v$ and $w$ are determined accordingly. Actually, $d$ can be any nonzero value.
} 

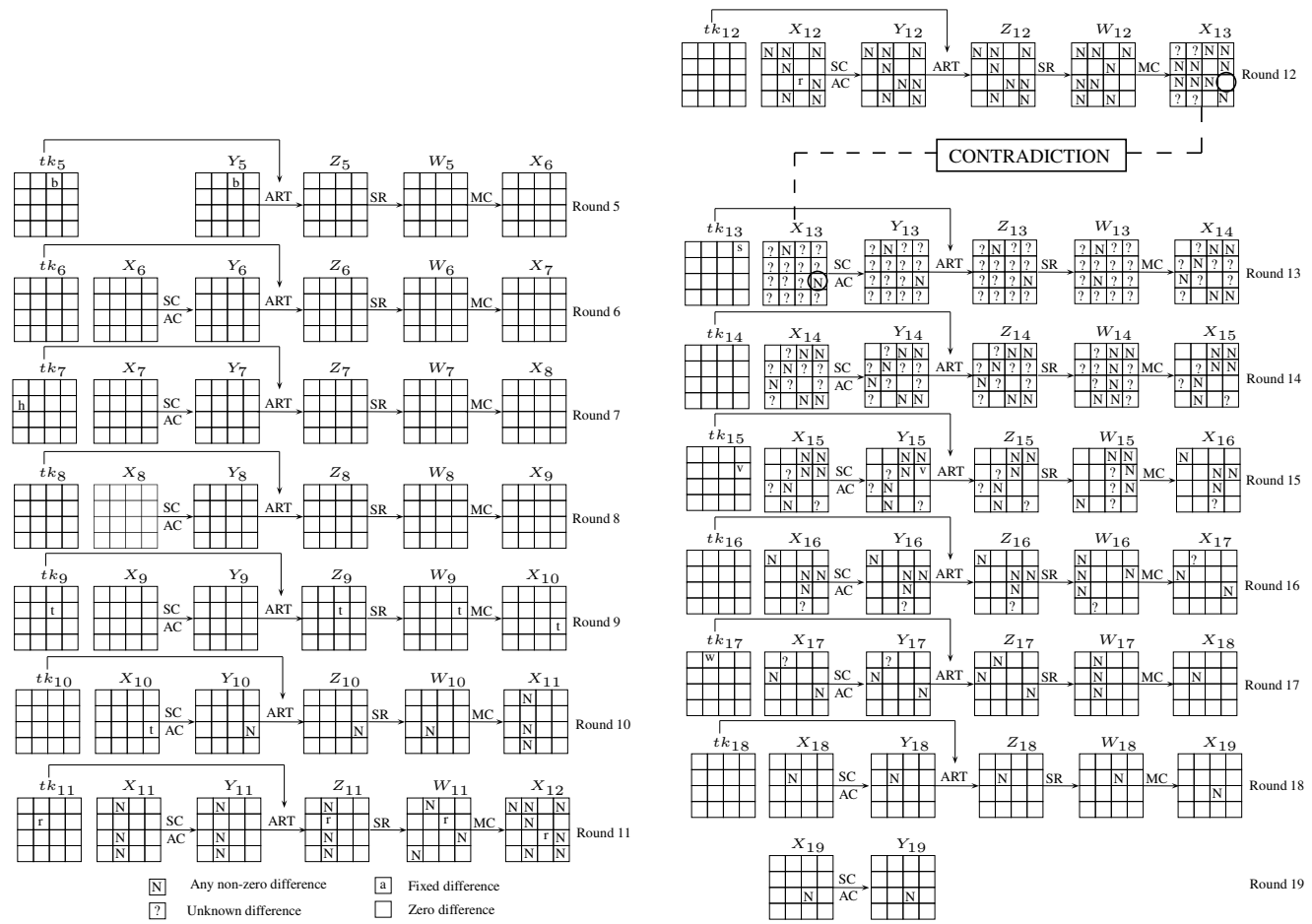

Figure 5: 14-round related-tweakey impossible distinguisher on SKINNY- $n$ - $2 n$.

$E^{\prime}$ under a pair of related-tweakeys and is used to attack $E=E_{f} \circ E^{\prime} \circ E_{b}$. Through $E_{b}^{-1}$ $\left(E_{f}\right), \Delta X(\Delta Y)$ is propagated to $\Delta_{\text {in }}\left(\Delta_{\text {out }}\right)$ with probability 1 . Let $c_{\text {in }}\left(c_{\text {out }}\right)$ be the number of bit conditions that need to be verified for $\Delta_{\text {in }} \rightarrow \Delta X\left(\Delta Y \leftarrow \Delta_{\text {out }}\right)$, and $k_{\text {in }}$ $\left(k_{\text {out }}\right)$ is the key information involved in $E_{b}\left(E_{f}\right)$.

Consider the two secret related-tweakey inputs to be - $(T K-1)^{1} \|(T K-2)^{1}$ and $(T K-$ $1)^{2} \|(T K-2)^{2}$. Consider further the difference $\Delta$ injected in round 1 subtweakey to be: $\Delta$

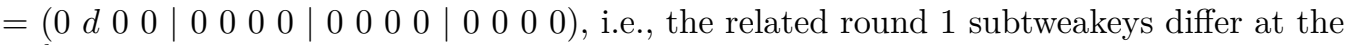
$2^{\text {nd }}$ cell-position and the difference $d$ is known to the attacker. The 14-round distinguisher is extended 4.5 rounds at the top and 4.5 rounds in the bottom to cover 9 rounds in the tweakey recovery phase as shown in Fig. 6 . Here, $E^{\prime}$ covers 14 rounds, $E_{f}\left(E_{b}\right)$ covers 4.5 (4.5) rounds, $c_{\text {in }}=\left|\Delta_{\text {in }}\right|=7 c, c_{\text {out }}=15 c,\left|\Delta_{\text {out }}\right|=16 c$ and $\left|k_{\text {in }} \cup k_{\text {out }}\right|=31 c$ where $c$ is the cell size.

As discussed in Section 2.3, the order of $A R T, S R$ and $M C$ operations in round 1 can be changed. Since, the design of SKINNY does not include a pre-whitening key, the input difference at $W_{1}$ (denoted as $P^{e q}$ in Fig. 6) can be considered as the plaintext difference and the equivalent plaintexts so obtained can be rolled back to compute the actual plaintexts. In the following discussion, we start our tweakey recovery attack at $W_{1}$ and call the inputs at this position as the plaintext inputs. In addition, we only pay attention to the values of subtweakeys and sometimes omit the effect of subtweakey differences for simplicity, since subtweakey differences are known to the attacker.

The steps of our attack are as follows:

Data collection Consider a pair of structures $S_{1}$ and $S_{2}$, where, each structure consists of $2^{\left|\Delta_{i n}\right|}=2^{7 c}$ plaintexts and for each plaintext pair $P_{1} \in S_{1} \& P_{2} \in S_{2}, P_{1} \oplus P_{2}=(0$ $0 N 0|N N 0 N| N 0 N 0 \mid 0 N 00)$, where $N$ denotes any cell value. The total number of possible plaintext pairs is $2^{2\left|\Delta_{i n}\right|}=2^{14 c}$. Invert back the plaintexts in $S_{1}$ and $S_{2}$ by one keyless round to get the original plaintexts. Encrypt the pool $S_{1}$ 
under $(T K-1)^{1} \|(T K-2)^{1}$ and the pool $S_{2}$ under $(T K-1)^{2} \|(T K-2)^{2}$ to obtain the corresponding ciphertexts. For each ciphertext pair, check whether $n-\left|\Delta_{\text {out }}\right|$ bits are zero or not and discard it if false. ${ }^{2}$ Generate $2^{x}$ such pair of structures and repeat this for each pair of structures. Note that, in the attack of SKINNY- $n-2 n$, more than one pair of structures are used and the case which requires less than one pair of structures is discussed latter. In total, $M=2^{x+2\left|\Delta_{i n}\right|-n+\left|\Delta_{\text {out }}\right|}=2^{x+14 c}$ plaintext pairs are expected to remain. This step requires a total of $2^{x+\left|\Delta_{i n}\right|+1}=2^{x+7 c+1}$ encryption calls.

Tweakey recovery For each of the $M$ pairs as shown in Fig. $6:^{3}$

(a) Compute $\Delta X_{23}[10,12,14]$ from the knowledge of the ciphertext pair values as computing these cells do not require any tweakey information. Due to $M C$ operation on the active nibbles of $\operatorname{Col}(1)$ and $\operatorname{Col}(3)$ of $W_{22}$, it can be seen that $\Delta X_{23}[0]=\Delta X_{23}[4]=\Delta X_{23}[12]$ and $\Delta X_{23}[6]=\Delta X_{23}[10] \oplus \Delta X_{23}[14]$ and thus can be determined. From this information, $X_{23}[0], Y_{23}[0]$ and thus $t k_{23}[0]$ can be found using Lemma 1 as $\Delta Y_{23}[0]$ is known from the ciphertexts. Similarly, the tweakey cells $t k_{23}[4,6]$ can also be derived. Guess $t k_{23}[1,2,3,5,7]$. Compute $Z_{22}$ and $\Delta Z_{22}[0-4,6-9,11-12,14-15]$. The time complexity of this step is $M \cdot 2^{5 c}$ one-round encryptions and a total of $M \cdot 2^{5 c}$ tests need to be done in the next step.

(b) Compute $\Delta X_{22}[8,11,12,14,15]$ from the knowledge of $Z_{22}$ and $\Delta Z_{22}$ known cells. Due to $M C$ operation on the active nibbles of $\operatorname{Col}(1,3,4)$ of $W_{21}$, $\Delta X_{22}[0,2,4,6,7]$ can be determined as - $\Delta X_{22}[0]=\Delta X_{22}[12], \Delta X_{22}[4]=$ $\Delta X_{22}[8] \oplus \Delta X_{22}[12], \Delta X_{22}[2]=\Delta X_{22}[6]=\Delta X_{22}[14]$ and $\Delta X_{22}[7]=\Delta X_{22}[11] \oplus$ $\Delta X_{22}[15]$. Use this information and the known differences of $\Delta Z_{22}$ to derive $t k_{22}[0,2,4,6,7]$ by using Lemma 1 . Guess $t k_{22}[1,3,5]$. Compute $Z_{21}$ and $\Delta Z_{21}$ as shown in Fig. 6 . The time complexity of this step is $M \cdot 2^{8 c}$ one-round encryptions and a total of $M \cdot 2^{8 c}$ tests need to be done in the next step.

(c) Compute $\Delta X_{21}[10,12,14]$ from the knowledge of $Z_{21}$ and $\Delta Z_{21}$ known cells. Check if $\Delta X_{21}[10]=\Delta X_{21}[14]$. This acts as one-cell filter. As $\Delta X_{21}[0]=$ $\Delta X_{21}[4]=\Delta X_{21}[12]$ and $\Delta X_{21}[2]=\Delta X_{21}[10]$, use this information and the known differences of $\Delta Z_{21}$ to derive $t k_{21}[0,2,4]$ by using Lemma 1 . At this stage, the attacker can uniquely determine $(T K-1)^{1}[0,1,7]$ and $(T K-2)^{1}[0,1,7]$ from the knowledge of $t k_{21}[0,2,4]$ and $t k_{23}[2,4,6]$. This helps her to determine $t k_{1}[0,1,7]$ and $t k_{3}[2]$. This step has a time complexity of $M \cdot 2^{8 c}$ one-round encryptions and the number of tests left for the next step is $M \cdot 2^{7 c}$.

(d) Since $t k_{1}^{e q}[0,1,4,5,8,12,13]$ is known from the previous steps, we can compute $X_{2}[0,1,4,5,8,12,13]$ and thus, $Y_{2}[0,1,4,5,8,12,13]$. From this, $\Delta Y_{2}[5,8]$ can be computed. Check if $\Delta Y_{2}[5]=\Delta Y_{2}[8]$ or not. This acts as a one-cell filter. Also, since $\Delta Y_{2}[2]=\Delta Y_{2}[8]$ (due to $M C^{-1}$ operation on $\operatorname{Col}(3)$ of $X_{3}$ ), use this information and the plaintext difference to derive $t k_{1}[2]$ by using Lemma 1.

\footnotetext{
${ }^{2}$ In this attack, this step is skipped as $n=\left|\Delta_{\text {out }}\right|$. In our 19-round attack on SKINNY- $n-n$, this step is not skipped.

${ }^{3}$ In Fig. 6, we have shown what kind of information in the state cells (only difference or only value or difference as well as value) is required to verify the differential path from $\Delta Y_{19} \rightarrow \Delta C$ and $\Delta Y_{5} \rightarrow \Delta P$. For example, consider rounds 19 to 23. During the key recovery phase, we proceed by collecting those key guesses for which the given ciphertext pair follows the differential trail from $\Delta Y_{19} \rightarrow \Delta C$ (represented by the greyed out cells). This is done by checking in each round whether $\Delta X_{i+1}$ leads to the required difference $\Delta W_{i}$ or not for $19 \leq i \leq 23$. Starting from $\Delta C$, to calculate $\Delta X_{i}$ in each round $i$, we need to know the difference and the state values in the active cells of the corresponding $\Delta Y_{i}$ 's. For computing the required state values of $Y_{i}^{\prime}$ 's in each round $i$, we need to know the state values of cells (which may not be differentially active) as well as the key values in the subsequent rounds (round $i+1$ till round 23) on which the $Y_{i}$ 's are dependent. In Fig.6, the struck out cells in the state cells represent that information.
} 


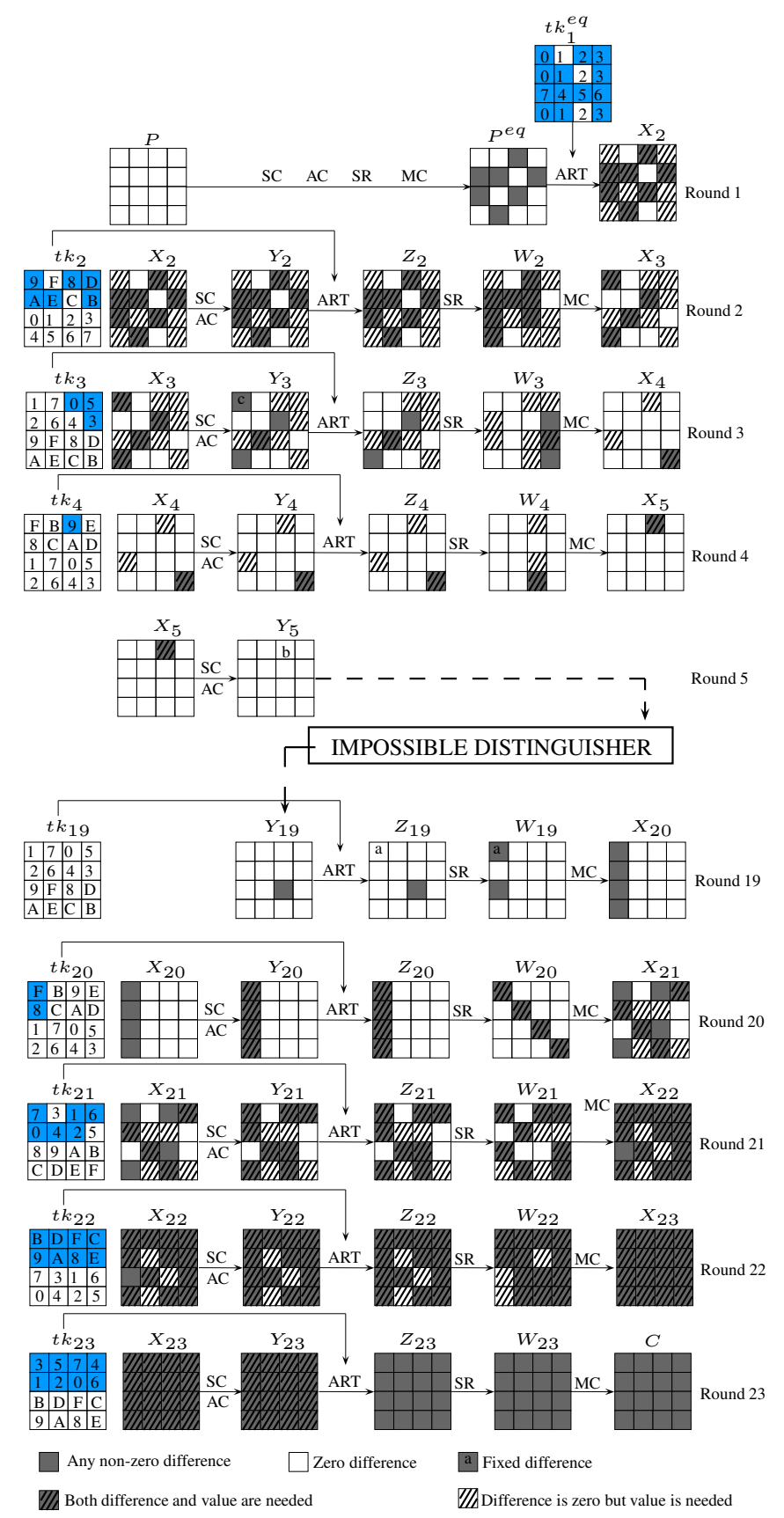

Figure 6: 23-round attack against SKINNY- $n-2 n$. The colored cells in each round tweakeys represent the cells, the values for which should be known to verify the differential path. During our attack, some of these tweakey cells are guessed and some of them are derived from the information that we have at each step of the attack.

At this stage, the attacker can uniquely determine $(T K-1)^{1}[2]$ and $(T K-2)^{1}[2]$ and compute $t k_{1}[2]$ and $t k_{21}[6]$. The time complexity of this step is $M \cdot 2^{7 c}$ and the number of tests left for the next step is $M \cdot 2^{6 c}$.

(e) Guess $t k_{21}[3,5]$. Compute $\Delta Z_{20}$ and $Z_{20}[0,4,8,12]$. Compute $\Delta X_{20}[8,12]$. 
Now, since $\Delta X_{20}[0]=\Delta X_{20}[12]$ and $\Delta X_{20}[4]=\Delta X_{20}[8] \oplus \Delta X_{20}[12]$ (due to MC operation on $\operatorname{Col}(1)$ of $\left.W_{19}\right), t k_{20}[0,4]$ can be derived using Lemma 1 . The knowledge of $\Delta X_{20}[4]$ also allows her to compute $\Delta W_{19}[0]$. Check if $\Delta W_{19}[0]=$ $a$ or not where, the difference $a$ is known to the attacker. We obtain a filter of one cell. At this stage, the attacker can uniquely determine $(T K-1)^{1}[4,6,8,15]$ and $(T K-2)^{1}[4,6,8,15]$. This allows her to compute $t k_{1}[4,6]$ and $t k_{2}[1,2]$. This step has a time complexity of $M \cdot 2^{8 c}$ one-round encryptions and the number of tests left after this step is $M \cdot 2^{7 c}$.

(f) Guess $t k_{1}[5]$ and compute $\Delta Y_{2}[10]$. Since, $\Delta Y_{2}[7]=\Delta Y_{2}[10]$ and from the plaintexts $\Delta X_{2}[7]$ is known, determine $X_{2}[7]$ and thus $t k_{1}^{e q}[7]=t k_{1}[3]$. At this stage, the attacker can uniquely determine $(T K-1)^{1}[3,5]$ and $(T K-2)^{1}[3,5]$ and thus, the full $t k_{1}^{e q}$ (and $t k_{1}$ ) is known to her. This allows her to compute $t k_{3}[3,7]$ and full $Y_{2}$ state (value as well as difference). As per the $M C^{-1}$ definition, $\Delta W_{2}[12]=\Delta X_{3}[0] \oplus \Delta X_{3}[12]$. Since, $\Delta W_{2}[12]\left(=\Delta Y_{2}[13]\right)$ and $\Delta X_{3}[12](=$ $\left.\Delta W_{2}[4]=\Delta Y_{2}[7]\right)$ are known to the attacker, she can compute $\Delta X_{3}[0]$. Since, $\Delta Y_{3}[0]=c$ is also known to her, $X_{3}[0]$ can be determined. Using $X_{3}[0], W_{2}[0]$ $=X_{3}[0] \oplus W_{2}[8] \oplus W_{2}[12]$ can be determined. This helps the attacker to derive $t k_{2}[0]$ (At this stage, the attacker can uniquely determine $(T K-1)^{1}[9]$ and $(T K-2)^{1}[9]$ and compute $\left.t k_{4}[2]\right)$. Further, $X_{3}[12]=W_{2}[0] \oplus W_{2}[8]$ can also be computed. This allows her to compute $Y_{3}[12]$ and $\Delta Y_{3}[12]$. It can be easily verified that $\Delta Y_{3}[12]=\Delta Y_{3}[6]=\Delta Y_{3}[9]$ (due to $M C^{-1}$ operation on $\operatorname{Col}(4)$ of $\left.X_{4}\right)$. It can also be observed that $\Delta X_{3}[6]=\Delta Y_{2}[5]$ and $\Delta X_{3}[9]=$ $\Delta Y_{2}[4]$ due to $M C^{-1}$ operation in Round 3. Thus, $X_{3}[6,9]$ and $Y_{3}[6,9]$ can be determined. Now, $X_{3}[6]=W_{2}[2]$ and $W_{2}[2]$ is already known to the attacker as $W_{2}[2]=Y_{2}[2] \oplus t k_{2}[2]$. Hence, a one-cell filter is obtained. Using $X_{3}[9]$, obtain $W_{2}[5]$ and derive $t k_{2}[4]$. Attacker can now uniquely determine $(T K-1)^{1}[10]$ and $(T K-2)^{1}[10]$. This step has a time complexity of $M \cdot 2^{8 c}$ one-round encryptions and the number of tests left after this step is $M \cdot 2^{7 c}$

(g) Compute $X_{3}[2]=W_{2}[2] \oplus W_{2}[10] \oplus W_{2}[14]$. Guess $t k_{2}[3,7]$. This allows the attacker to compute $X_{3}[3,7,8,15]$. Through this, she can then further compute $X_{4}[2,15], \Delta X_{4}[15]$ and $\Delta Y_{4}[15]$. Now, $\Delta Y_{4}[15]=\Delta X_{5}[2]$ (due to $M C^{-1}$ operation on $\operatorname{Col}(3)$ of $\left.X_{5}\right)$. As, $\Delta Y_{5}[2]=b$ is already known to her, using S-box equations she can compute $X_{5}[2]$. Now, $S\left(X_{4}[8]\right)=X_{5}[2] \oplus S\left(X_{4}[2]\right) \oplus$ $t k_{4}[2] \oplus S\left(X_{4}[15]\right)$. Since, the right hand side of the equation is known to the attacker, she can determine $X_{4}[8]$. This allows her to compute $W_{3}[8]$ and further $X_{3}[10]$. Once $X_{3}[10]$ is known, $W_{2}[6]$ can be determined which in turn helps in calculating $t k_{2}[5]$. The time complexity of this step is $M \cdot 2^{9 c}$ and the number of tests verifying the impossible distinguisher is $M \cdot 2^{9 c}$, i.e., for each pair there are $2^{9 c} 31$-cell keys on average that verify the impossible distinguisher and are wrong.

The time complexity of analyzing $M$ pairs is $M \cdot 2^{9 c}$. The total number of tweakeys left is:

$$
T K_{\text {rem }}=2^{\left|k_{\text {in }} \cup k_{\text {out }}\right|}\left(1-2^{-\left(c_{\text {in }}+c_{\text {out }}\right)}\right)^{M}=2^{31 c}\left(1-2^{-22 c}\right)^{2^{x+14 c}}
$$

Suppose $\left(1-2^{-\left(c_{\text {in }}+c_{\text {out }}\right)}\right)^{M}=2^{-g}, 1<g \leq\left|k_{\text {in }} \cup k_{\text {out }}\right|$, which means $g$-bit key material is recovered, then we have $M=2^{c_{\text {in }}+c_{\text {out }}} g \ln 2$ since $\left(1-2^{-\left(c_{\text {in }}+c_{\text {out }}\right)}\right)^{M} \approx$ $e^{-M 2^{-\left(c_{\text {in }}+c_{\text {out }}\right)}}$. We also know that, $M=2^{x+2\left|\Delta_{\text {in }}\right|-n+\left|\Delta_{\text {out }}\right|}$, therefore, $2^{x}=$ $2^{c_{i n}+c_{\text {out }}+n-2\left|\Delta_{i n}\right|-\left|\Delta_{\text {out }}\right|} \times g \ln 2$, and thus the data complexity is $D=2^{x+\left|\Delta_{i n}\right|+1}=$ $2^{c_{\text {in }}+c_{\text {out }}+n+1-\left|\Delta_{\text {in }}\right|-\left|\Delta_{\text {out }}\right|} g \ln 2$.

Brute force For the tweakeys that remain, we guess the remaining tweakey cells (1 cell) and exhaustively search the $T K_{r e m} \times 2^{c}=2^{2 n-g}$ tweakeys to find the correct tweakey. 
Attack Complexities. The attack described above requires a data complexity of $D=$ $2^{c_{\text {in }}+c_{\text {out }}+n+1-\left|\Delta_{\text {in }}\right|-\left|\Delta_{\text {out }}\right|} g \ln 2=2^{n+1-c} g \ln 2$ chosen plaintexts. The total time complexity is the summation of the time consumption of all the steps:

$$
T=D+M \cdot 2^{9 c}+T K_{r e m} \cdot 2^{c} .
$$

The memory complexity is the storage for one structure and wrong keys. For $c=4$, we set $g=4$, then $D=2^{62.47}, M \cdot 2^{9 c}=2^{125.47}, T K_{r e m} \cdot 2^{c}=2^{124}, T=2^{125.91}$ and the memory complexity is $2^{124}$. For $c=8$, we set $g=16$, then $D=2^{124.47}, M \cdot 2^{9 c}=2^{251.47}$, $T K_{\text {rem }} \cdot 2^{c}=2^{240}, T=2^{251.47}$ and the memory complexity is $2^{248}$.

Attack where tweak is used. Suppose a $w$-bit tweak is used. The tweak is loaded into the first $w$ bits of the first tweakey state $T K-1$, followed by the key material. For $0 \leq w \leq 9 c$, we have $\left|k_{\text {in }} \cup k_{\text {out }}\right|=2 n-w-c<2 n-w$ and the above attack still has a time complexity below $2^{2 n-w}$, so our 23-round attack on SKINNY- $n$ - $2 n$ is valid in such cases. For $9 c<w \leq 12 c$, we still have $\left|k_{\text {in }} \cup k_{\text {out }}\right|=2 n-w-c<2 n-w$, but the time complexity of the tweakey recovery phase is higher than $2^{2 n-w}$. For $w=9 c$, the results are as follows. For $c=4$, we set $g=3$, then $D=2^{62.06}, T=2^{90.03}$ and the memory complexity is $2^{88}$. For $c=8$, we set $g=6$, then $D=2^{123.06}, T=2^{179.03}$ and the memory complexity is $2^{176}$.

For SKINNY- $n-n$ and SKINNY- $n-3 n$, our results are as shown in Table 3. For more information regarding the impossible distinguishers under TK1 and TK3, we refer the readers to Appendix A. The 19-round attack of SKINNY- $n$ - $n$ and the 27 -round attack of SKINNY- $n-3 n$ is presented in Appendix B.

Table 3: Results of related-tweakey impossible differential attack on SKINNY

\begin{tabular}{lrccccccc}
\hline Model & Version & \#Rounds & $R_{d}$ & $R_{b}$ & $R_{f}$ & Data & Time & Memory \\
\hline TK1 & $64-64$ & 19 & 12 & 4.5 & 2.5 & $2^{61.47}$ & $2^{63.03}$ & $2^{56}$ \\
& $128-128$ & 19 & 12 & 4.5 & 2.5 & $2^{122.47}$ & $2^{124.60}$ & $2^{112}$ \\
\hline TK2 & $64-128$ & 23 & 14 & 4.5 & 4.5 & $2^{62.47}$ & $2^{125.91}$ & $2^{124}$ \\
& $128-256$ & 23 & 14 & 4.5 & 4.5 & $2^{124.47}$ & $2^{251.47}$ & $2^{248}$ \\
\hline TK3 & $64-192$ & 27 & 16 & 6.5 & 4.5 & $2^{63.53}$ & $2^{189}$ & $2^{184}$ \\
& $128-384$ & 27 & 16 & 6.5 & 4.5 & $2^{126.03}$ & $2^{378}$ & $2^{368}$ \\
\hline
\end{tabular}

Attack where less than one pair of structures is used. In our 23-round attack on SKINNY- $n$ - $2 n$, a total of $M \cdot 2^{n-\left|\Delta_{\text {out }}\right|}$ plaintext- ciphertext pairs were obtained (out of which $M$ ciphertext pairs were left after ciphertext sieving) and $M \cdot 2^{n-\left|\Delta_{\text {out }}\right|}>2^{2\left|\Delta_{\text {in }}\right|}$. Therefore, more than one structure was required to carry out the attack. However, in some cases, $M \cdot 2^{n-\left|\Delta_{\text {out }}\right|} \leq 2^{2\left|\Delta_{i n}\right|}$ (e.g., in case of our 27-round attack on SKINNY$n-3 n$ discussed in Appendix B) and thus one structure is enough. In this case, $D=$ $2 \sqrt{M \cdot 2^{n-\left|\Delta_{\text {out }}\right|}}$. In a nutshell,

$$
D= \begin{cases}2^{c_{i n}+c_{\text {out }}+n+1-\left|\Delta_{\text {in }}\right|-\left|\Delta_{\text {out }}\right|} g \ln 2 & \text { if } M \cdot 2^{n-\left|\Delta_{\text {out }}\right|}>2^{2\left|\Delta_{i n}\right|} \\ 2 \sqrt{2^{c_{i n}+c_{\text {out }}+n-\left|\Delta_{\text {out }}\right|} g \ln 2} & \text { if } M \cdot 2^{n-\left|\Delta_{\text {out }}\right|} \leq 2^{2\left|\Delta_{i n}\right|}\end{cases}
$$

In the impossible distinguishers constructed in $\left[\mathrm{ABC}^{+} 17\right]$, the impossible differentials always start and end with a fully passive state in the distinguisher construction phase. Therefore, $c_{i n}=\left|\Delta_{\text {in }}\right|$ and $c_{\text {out }}=\left|\Delta_{\text {out }}\right|$ for these distinguishers. Then, the above formula 
of data complexity can be simplified as:

$$
D= \begin{cases}2^{n+1} g \ln 2 & \text { if }\left|\Delta_{i n}\right|<n+\log _{2} g \ln 2 \\ 2 \sqrt{2^{c_{i n}+n} g \ln 2} & \text { if }\left|\Delta_{i n}\right| \geq n+\log _{2} g \ln 2\end{cases}
$$

From this formula, it is thus learnt that the data complexity will always be beyond codebook, i.e., $D>2^{n}$ for the impossible differential attacks presented in $\left[\mathrm{ABC}^{+} 17\right]$.

\section{Truncated Related-Tweakey Differential Trails and Rect- angle Attacks}

In the last section, we constructed impossible distinguishers by connecting two truncated differential trails which propagate forward and backward with probability 1 under one related-tweakey. In this section, boomerang (rectangle) distinguishers are constructed by combining two truncated differential trails which propagate forward and backward with probability 1 under two distinct related-tweakeys.

This section first gives a brief introduction of boomerang attacks and rectangle attacks, and then presents rectangle distinguishers of SKINNY based on which 17, 22 and 27 rounds of SKINNY can be attacked under TK1, TK2 and TK3 respectively.

\subsection{Boomerang and Rectangle Attack}

Boomerang attack, proposed by David Wagner in FSE 1999 [Wag99], allows an attacker to concatenate two short differential trails. This proves beneficial in cases where long differential trails have a very low probability or it is difficult to search a long differential trail. In the basic setting of the attack, the block cipher is treated as a cascade of two sub-ciphers $E_{0}$ and $E_{1}$, each having a high probability short differential of its own. These differentials are then combined in a chosen plaintext and ciphertext attack setting to first construct a boomerang distinguisher and then use the distinguisher to recover the secret key.

Suppose $E_{0}$ covers the first $l$ rounds of encryption and $E_{1}$ covers the rest $(r-l)$ rounds of encryption. Let us further suppose, there exists a differential $\alpha \rightarrow \beta$ through $E_{0}$ with a high probability $p$. Similarly, there exists a differential $\delta \rightarrow \gamma$ through $\left(E_{1}\right)^{-1}$ which has a high probability $q$. The boomerang attack then proceeds as follows:

1. Consider two plaintexts $X, Y$ such that $Y=X \oplus \alpha$. Obtain their corresponding ciphertexts $X^{\prime \prime}, Y^{\prime \prime}$ respectively.

2. The probability that $E_{0}(X) \oplus E_{0}(Y)=X^{\prime} \oplus Y^{\prime}=\beta$ is p.

3. Obtain, $Z^{\prime \prime}=X^{\prime \prime} \oplus \delta$ and $W^{\prime \prime}=Y^{\prime \prime} \oplus \delta$. If we apply $\left(E_{1}\right)^{-1}$ to each of the pairs $\left(X^{\prime \prime}, Z^{\prime \prime}\right)$ and $\left(Y^{\prime \prime}, W^{\prime \prime}\right)$, then with probability $\mathbf{q}^{2},\left(E_{1}\right)^{-1}\left(X^{\prime \prime}\right) \oplus\left(E_{1}\right)^{-1}\left(Z^{\prime \prime}\right)=$ $X^{\prime} \oplus Z^{\prime}=\gamma$ and $\left(E_{1}\right)^{-1}\left(Y^{\prime \prime}\right) \oplus\left(E_{1}\right)^{-1}\left(W^{\prime \prime}\right)=Y^{\prime} \oplus W^{\prime}=\gamma$.

4. Then, the following statement holds true: With probability $\mathbf{p q}^{2},\left(E_{1}\right)^{-1}\left(Z^{\prime \prime}\right) \oplus$ $\left(E_{1}\right)^{-1}\left(W^{\prime \prime}\right)=\beta$. This is because $\left(E_{1}\right)^{-1}\left(Z^{\prime \prime}\right) \oplus\left(E_{1}\right)^{-1}\left(W^{\prime \prime}\right)=Z^{\prime} \oplus W^{\prime}=\left(Z^{\prime} \oplus\right.$ $\left.X^{\prime}\right) \oplus\left(X^{\prime} \oplus Y^{\prime}\right) \oplus\left(Y^{\prime} \oplus W^{\prime}\right)=\gamma \oplus \beta \oplus \gamma=\beta$.

5. Thus, with probability $\mathbf{p}^{\mathbf{2}} \mathbf{q}^{2}, E^{-1}\left(Z^{\prime \prime}\right) \oplus E^{-1}\left(W^{\prime \prime}\right)=Z \oplus W=\alpha$.

6. Now if, $(\mathbf{p q})>2^{-n / 2}$, then a valid distinguisher is constructed. This is because, for a random permutation, the expected probability that $Z \oplus W=\alpha$ is $2^{-n}$. 
Therefore, if $\mathbf{p}^{\mathbf{2}} \mathbf{q}^{\mathbf{2}}$ is sufficiently large, then the boomerang distinguisher can effectively distinguish between $E(\cdot)$ and a randomly chosen permutation, given a sufficient number of adaptive chosen plaintexts and ciphertexts. The plaintexts $(X, Y, Z, W)$ together are termed as a quartet and satisfy the following property:

$$
X \oplus Y \oplus Z \oplus W=0 .
$$

The basic boomerang attack explained above requires adaptive chosen plaintexts and ciphertexts. Later, Kelsey et al. [KKS00] developed amplified boomerangs which are pure chosen-plaintext attacks. In the case of amplified boomerang attacks, the attacker chooses certain amount of plaintext pairs and lets the oracle encrypt them. Any two pairs form a quartet $(X, Y, Z, W)$, and the difference $\gamma$ before $E_{1}$ holds with probability $2^{-n}$ for a quartet. Thus one can expect a right quartet where $X^{\prime \prime} \oplus Z^{\prime \prime}=Y^{\prime \prime} \oplus W^{\prime \prime}=\delta$ with probability $2^{-n} p^{2} q^{2}$. For a random permutation the expected probability is $2^{-2 n}$, so if $2^{-n} p^{2} q^{2}>2^{-2 n}$, a distinguisher can be constructed. In [BDK01], Biham et al. made further improvements which allow any value of $\beta$ and $\gamma$ to occur as long as $\beta \neq \gamma$ and renamed the attack as rectangle attack. As a result, the probability of the right quartet is increased to $2^{-n} \hat{p}^{2} \hat{q}^{2}$, where $\hat{p}=\sqrt{\Sigma_{i} \operatorname{Pr}^{2}\left(\alpha \rightarrow \beta_{i}\right)}$ and $\hat{q}=\sqrt{\Sigma_{j} \operatorname{Pr}^{2}\left(\gamma_{j} \rightarrow \delta\right)}$.

\subsection{Related-Tweakey Rectangle Distinguishers}

Our distinguisher takes the advantage of the differential cancellation behavior of the tweakey schedule to cover as many rounds as possible. Fig. 7 is a description of the generalized related-tweakey rectangle distinguisher.

For the sake of generalization, the distinguisher begins at round $R$ and the subtweakey difference $a$ is constant. As shown in Fig. 7, an input difference will transform to any possible output difference which is denoted by $N$. The notation ? means that we are not sure whether the cell is active or not. There is only one active tweakey cell in both the upper and the lower truncated differential trails. In the following discussion we take SKINNY-64 as an example, where each cell is a nibble.

In a typical rectangle distinguisher, usually, two differential trails are constructed, i.e., $\alpha \rightarrow \beta$ and $\gamma \leftarrow \delta$. Note that in the related-tweakey case, we specify a tweakey difference $\Delta_{1}$ for the upper trail $\alpha \rightarrow \beta$ and a tweakey difference $\Delta_{2}$ for the lower trail $\gamma \leftarrow \delta$. The position of the active tweakey cell is chosen by the rule that the truncated differential trail from round $R+2 z$ to round $R+2 z+3$ is the optimal differential trail as well as the truncated differential trail from round $R+2 z+7$ to round $R+2 z+4$.

The $\alpha \stackrel{\Delta_{1}}{\longrightarrow} \beta$ differential trail begins with a state difference $\alpha$ that contains one active cell in a carefully chosen position relating to the key state permutation. At Round $R$, a tweakey difference of one cell position is introduced to cancel the state difference. Therefore, we will have $2 z-1$ rounds of fully non-active internal state in the subsequent encryption process, e.g., we have 5 rounds of fully non-active internal states for TK3.

At round $R+2 z$, the key state introduces a cell difference $a$ to the internal states with the corresponding related-tweakey difference. The introduced internal difference continues to diffuse for four rounds according to the round function of SKINNY. At round $R+2 z+2$, another tweakey difference $a$ is introduced, which complicates the diffusion of the difference pattern through rounds.

Similarly, at the bottom of the distinguisher the input difference $\delta$ (with the same value as the tweakey difference $a$ ) of the differential trail $\gamma \stackrel{\Delta_{2}}{\longleftarrow} \delta$ is cancelled out by the tweakey difference $\Delta_{2}$ which results in $2 z-1$ rounds of fully inactive internal states. At round $R+2 z+7$, difference $a$ is inserted by the subtweakey difference and continues to propagate for 4 rounds just as in the upper trail $\alpha \stackrel{\Delta_{1}}{\longrightarrow} \beta$.

A rectangle distinguisher will work as long as the two upper trails $\alpha \stackrel{\Delta_{1}}{\longrightarrow} \beta$ as well as the two lower trails $\gamma \stackrel{\Delta_{2}}{\longleftarrow} \delta$ agree with each other (note that $\beta \neq \gamma$ ). Here we denote the 


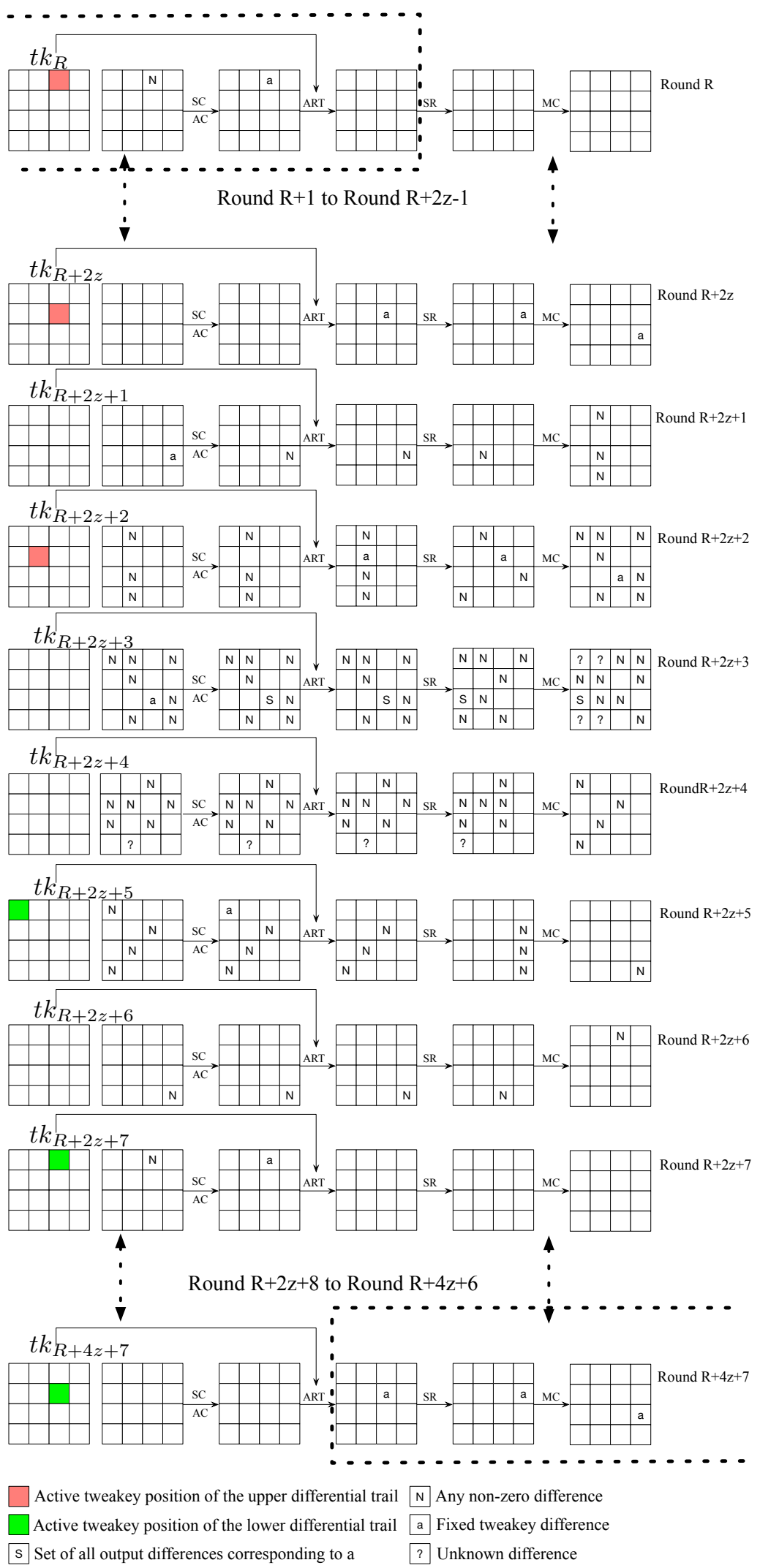

Figure 7: Description of the generalized related-tweakey rectangle distinguisher 
two upper trails as $\alpha \stackrel{\Delta_{1}}{\longrightarrow} \beta_{1}$ and $\alpha \stackrel{\Delta_{1}}{\longrightarrow} \beta_{2}$ respectively. Similarly, we denote the two lower trails as $\gamma_{1} \stackrel{\Delta_{2}}{\longleftarrow} \delta$ and $\gamma_{2} \stackrel{\Delta_{2}}{\longleftarrow} \delta$. Thus, a rectangle distinguisher will succeed as long as $\beta_{1}=\beta_{2}$ and $\gamma_{1}=\gamma_{2}$ while $\beta \neq \gamma$ are satisfied.

Instead of considering specific differential trails with high probability, we focus on all possible differential trails, which means the output difference $\beta$ of the upper trail $\alpha \stackrel{\Delta_{1}}{\longrightarrow} \beta$ can take any possible value. We employ the same strategy when analyzing the differential probability of the lower trail $\gamma \stackrel{\Delta_{2}}{\longleftarrow} \delta$, i.e., $\gamma_{1}$ and $\gamma_{2}$ can be any possible values.

Thus, the probability of the upper trail $\alpha \stackrel{\Delta_{1}}{\longrightarrow} \beta$ of the related-tweakey rectangle distinguisher is ${ }^{4} \sum_{\beta_{1}, \beta_{2}} P_{r}\left(\alpha \stackrel{\Delta_{1}}{\longrightarrow} \beta_{1}\right) \cdot P_{r}\left(\alpha \stackrel{\Delta_{1}}{\longrightarrow} \beta_{2}\right) \cdot P_{r}\left(\beta_{1}=\beta_{2}\right)=\left(2^{-3.9}\right)^{7} \cdot 2^{-2.4}=2^{-29.7}$. Similarly, the probability of the lower trail $\gamma \stackrel{\Delta_{2}}{\longleftarrow} \delta$ of the related-tweakey rectangle distinguisher is $\sum_{\gamma_{1}, \gamma_{2}} P_{r}\left(\gamma_{1} \stackrel{\Delta_{2}}{\longleftarrow} \delta\right) \cdot P_{r}\left(\gamma_{2} \stackrel{\Delta_{2}}{\longleftarrow} \delta\right) \cdot P_{r}\left(\gamma_{1}=\gamma_{2}\right)=\left(2^{-3.9}\right)^{6} \cdot 2^{-4}=2^{-27.4}$. The total probability of the related-tweakey rectangle distinguisher would be $2^{-29.7} \cdot 2^{-27.4}=$ $2^{-57.1}$. To be consistent with conventional notations, we define $\hat{p}^{2} \hat{q}^{2}=\sum_{\beta_{1}, \beta_{2}} P_{r}\left(\alpha \stackrel{\Delta_{1}}{\longrightarrow}\right.$ $\left.\beta_{1}\right) \cdot P_{r}\left(\alpha \stackrel{\Delta_{1}}{\longrightarrow} \beta_{2}\right) \cdot P_{r}\left(\beta_{1}=\beta_{2}\right) \cdot \sum_{\gamma_{1}, \gamma_{2}} P_{r}\left(\gamma_{1} \stackrel{\Delta_{2}}{\longleftarrow} \delta\right) \cdot P_{r}\left(\gamma_{2} \stackrel{\Delta_{2}}{\longleftarrow} \delta\right) \cdot P_{r}\left(\gamma_{1}=\gamma_{2}\right)$.

The generalized related-tweakey distinguisher works for both SKINNY-64 and SKINNY128 although we need to adjust the probability of the distinguisher for distinctive versions in use, which is an easy work. Particularly, the distinguisher is more effective on larger tweak size, e.g., the distinguisher consists of 19 rounds for TK3 while has only 15 rounds for TK2.

Discussion. In order to take full advantage of the tweakey differential cancellation property, the position of the active tweakey cell is determined cautiously taking into account two main factors. Firstly, the tweakey difference ought to offset the state difference at the beginning of the distinguisher, resulting in $2 z-1$ rounds of fully non-active rounds. Secondly, the truncated differential trail formed due to the tweakey difference should have at most 8 active cell positions at the meeting point, otherwise, the distinguisher won't work. With these requirements, we searched all the possible active cell positions of the tweakey and found 5 positions for the upper truncated differential trail and 4 positions for the lower truncated differential trail that satisfy our requirements.

The above distinguisher is constructed using one of the optimal truncated trails covering maximum number of rounds and having minimal probability. Moreover, the chosen active tweakey position also ensures that the truncated differential trails in the extended rounds of the tweakey recovery process are optimal (enabling maximum rounds to be covered), indicating that it is the best distinguisher under all the considertations.

Experimental verification of the distinguisher. The validity of our distinguishers is verified with an experiment on SKINNY-64-128 which aims at finding a right quartet for the 14-round boomerang distinguisher with probability $2^{-40}$. The result shows that averagely there is one right quartet among $2^{35.7}$ trials. The experiment not only verifies the correctness of our distinguishers but also demonstrates that the probability of our distinguishers are not overestimated. One of the right quartets obtained and more details of the experiment is shown in Appendix C.

\footnotetext{
${ }^{4}$ We use $1 / 15=2^{-3.9}$ to compute the probability of two output difference (i.e., $\beta_{1}$ and $\beta_{2}$ ) of the truncated differential to collide rather than a general $2^{-4}$. In the attack, the actual tweakey difference value at round $R+2 z+3$ results in 6 rather than 8 possible output differences. Thus, the corresponding probability would be $1 / 6=2^{-2.4}$ rather than $1 / 8=2^{-3}$.
} 


\subsection{Key Recovery Algorithm Based on Related-Tweakey Rectangle Distinguishers}

Our algorithm for using related-tweakey rectangle distinguisher in a key recovery attack is adapted from Biham et al.'s algorithm [BDK02] where in their rectangle key recovery attack there is no key difference. Note that this adapted key recovery algorithm is applicable to other block ciphers under related-tweakey (-key) model as long as the key schedule is linear.

We continue to use the notations and conventions of boomerang and rectangle attacks. The cipher $E$ is expressed as $E=E_{f} \circ E^{\prime} \circ E_{b}$ where, $E^{\prime}=E_{1} \circ E_{0}$ is the rectangle distinguisher and $E_{b}$ and $E_{f}$ are the rounds extended in the backward and forward direction of the distinguisher respectively (as shown in Fig. 8).

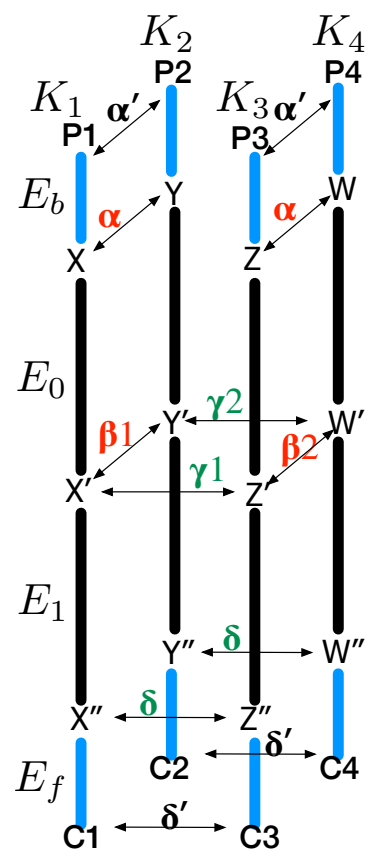

Figure 8: Key recovery model of related-tweakey rectangle attack

In a related-tweakey rectangle attack, the quartet of $\left(P_{1}, P_{2}, P_{3}, P_{4}\right)$ as plaintexts and $\left(C_{1}, C_{2}, C_{3}, C_{4}\right)$ as ciphertexts is encrypted with four related-tweakeys $\left(K_{1}, K_{2}, K_{3}, K_{4}\right)$ which are related to each other through specific tweakey differences. Let the input difference $\alpha$ of the distinguisher diffuse in the backward direction of the cipher $E$ over several rounds (which is $E_{b}$ section of $E$ ) with the related-tweakey difference $\Delta_{1}$ and all the possible output differences $\alpha^{\prime}$ (which are the actual plaintext differences) corresponding to $\alpha$ be stored in a set $U_{b}$. Likewise, let the output difference $\delta$ of the distinguisher diffuse to several rounds in the forward direction of the cipher with a related-tweakey difference $\Delta_{2}$ and all the possible output differences $\delta^{\prime}$ (which are the actual ciphertext differences) be stored in a set $U_{f}$.

Before we continue we introduce some additional notations. Let $V_{b}$ be the space spanned by the values in $U_{b}$. Let $r_{b}=\log _{2}\left|V_{b}\right|$ and $t_{b}=\log _{2}\left|U_{b}\right|$. Let $m_{b}$ be the the number of subtweakey bits involved in $E_{b}$ that affect the plaintext difference while encrypting plaintext pairs whose difference after $E_{b}$ is $\alpha$. Similarly, we define $U_{f}, V_{f}, r_{f}, t_{f}, m_{f}$ for $E_{f}$, i.e., $U_{f}$ is the set of ciphertext differences that may cause a difference $\delta$ before $E_{f}$ under the tweakey difference $\Delta_{2}, V_{f}$ is the space spanned by values of $U_{f}$ and $r_{f}=\log _{2}\left|V_{f}\right|, t_{f}=\log _{2}\left|U_{f}\right|$. 
$m_{f}$ is the number of subtweakey bits involved in $E_{f}$ that affect the ciphertext difference while decrypting pairs whose difference before $E_{f}$ is $\delta$. Suppose the master key is of $k$ bits.

Our algorithm proceeds as follows:

1. Let $K_{1}$ be the secret key and $K_{2}=K_{1} \oplus \Delta_{1}, K_{3}=K_{1} \oplus \Delta_{2}$ and $K_{4}=K_{1} \oplus \Delta_{1} \oplus \Delta_{2}$. Create $y=\sqrt{s} \cdot 2^{n / 2-r_{b}} / \hat{p} \hat{q}$ structures of $2^{r_{b}}$ plaintexts each, where $s$ is the expected number of right quartets. Encrypt these $y$ structures with $K_{1}, K_{2}$ respectively. Similarly, create $y$ structures of $2^{r_{b}}$ plaintexts each and encrypt these $y$ structures with $K_{3}, K_{4}$ respectively.

2. Initialize a list of $2^{m_{b}+m_{f}}$ counters, each of which corresponds to a $\left(m_{b}+m_{f}\right)$-bit subkey guess.

3. Under each key there are $M=y 2^{r_{b}}$ ciphertexts. Denote the ciphertext sets under $K_{i}, i \in\{1,2,3,4\}$ by $L_{i}, i \in\{1,2,3,4\}$. Process $\left(L_{1}, L_{3}\right)$ and $\left(L_{2}, L_{4}\right)$ independently. Insert the $L_{1}$ ciphertexts into a hash table $H_{1}$ according to the $n-r_{f}$ ciphertext bits that are set to 0 in $V_{f}$. Then for each ciphertext in $L_{3}$, try to find collisions of this ciphertext and ciphertexts in $H_{1}$. If a ciphertext pair from $L_{1}$ and $L_{3}$ agrees on the $n-r_{f}$ bits, check whether the ciphertext difference is in $U_{f}$. Do the same for $\left(L_{2}, L_{4}\right)$.

4. For each collision $\left(C_{1}, C_{3}\right) \in L_{1} \times L_{3}$ that is obtained from Step 3, denote $C_{i}$ 's structure under $K_{j}$ by $S_{C_{i}}^{K_{j}}$ and attach to $C_{1}$ the index of $S_{C_{3}}^{K_{3}}$. For each collision $\left(C_{2}, C_{4}\right) \in L_{2} \times L_{4}$, attach to $C_{2}$ the index of $S_{C_{4}}^{K_{4}}$.

5. In each structure $S$ under $K_{1}, K_{2}$, we search for two ciphertexts $C_{1} \in S^{K_{1}}, C_{2} \in S^{K_{2}}$ which are attached to some other structures under $K_{3}$ and $K_{4}$ respectively. Once we find such a pair, we first check that structures that $C_{1}$ and $C_{2}$ are attached to are same or not and whether the corresponding plaintext difference $P_{1} \oplus P_{2}$ is in $U_{b}$. We also check the difference of the plaintexts which $P_{1}$ and $P_{2}$ are related to.

6. For all the quartets that pass Step 5 , denote by $\left(P_{1}, P_{2}, P_{3}, P_{4}\right)$ the plaintexts of a quartet and by $\left(C_{1}, C_{2}, C_{3}, C_{4}\right)$ the corresponding ciphertexts under $\left(K_{1}, K_{2}, K_{3}, K_{4}\right)$. Increment the counters of $\left(m_{b}+m_{f}\right)$-bit subkeys which satisfy that $E_{b}^{K_{1}}\left(P_{1}\right) \oplus$ $E_{b}^{K_{2}}\left(P_{2}\right)=E_{b}^{K_{3}}\left(P_{3}\right) \oplus E_{b}^{K_{4}}\left(P_{4}\right)=\alpha$ and $\left(E_{f}^{K 1}\right)^{-1}\left(C_{1}\right) \oplus\left(E_{f}^{K 3}\right)^{-1}\left(C_{3}\right)=\left(E_{f}^{K 2}\right)^{-1}\left(C_{2}\right)$ $\oplus\left(E_{f}^{K 4}\right)^{-1}\left(C_{4}\right)=\delta$.

7. Output the subkeys corresponding to top $2^{m_{b}+m_{f}-h}$ hits, i.e., we recover $h$ bits information of the key in previous steps. We guess the remaining $k-m_{b}-m_{f}$ tweakey bits and exhaustively search the $2^{k-h}$ keys to find the correct one.

The data complexity of the attack is $D=4 M$ chosen plaintexts. The time complexity of Step 1 is $D$ encryptions. The time complexity of Step 2 is only $2^{m_{b}+m_{f}}$ memory access using suitable data structures.

Step 3 requires $2 M$ memory access for the insertion of both $\left(L_{1}, L_{3}\right)$ and $\left(L_{2}, L_{4}\right)$ and thus $4 M$ memory accesses in total. The total number of colliding pairs for both $\left(L_{1}, L_{3}\right)$ and $\left(L_{2}, L_{4}\right)$ is $M^{2} \cdot 2^{r_{f}-n}$. We keep all the $2^{t_{f}}$ values of $U_{f}$ in a hash table $H_{2}$, and thus, the check requires one memory access for each colliding pair obtained from $H_{1}$. This requires $M^{2} \cdot 2^{r_{f}-n}$ memory accesses for both $\left(L_{1}, L_{3}\right)$ and $\left(L_{2}, L_{4}\right)$. Out of the $2^{r_{f}}$ possible differences for a colliding pair, only $2^{t_{f}}$ differences are in $U_{f}$. In total, about $M^{2} \cdot 2^{t_{f}-n}$ colliding pairs are in $U_{f}$ for both $\left(L_{1}, L_{3}\right)$ and $\left(L_{2}, L_{4}\right)$. The time complexity of this step is $4 M+2 \cdot M^{2} \cdot 2^{r_{f}-n}$ memory accesses.

Step 4 requires one memory access for each pair remained after Step 3. The time complexity is $2 \cdot M^{2} \cdot 2^{t_{f}-n}$ memory accesses. 
Step 5 searches for possible quartets. In structures under $K_{1}$ (or $K_{2}$ ), there are $M^{2} \cdot 2^{t_{f}-n}$ attachments which distribute over $y$ structures. Therefore in each structure $K_{1}$ (or $K_{2}$ ), we have about $M^{2} \cdot 2^{t_{f}-n} / y=M \cdot 2^{t_{f}+r_{b}-n}$ attachments on average. In the same structure under $K_{1}$ and $K_{2}$, there are $\left(M \cdot 2^{t_{f}+r_{b}-n}\right)^{2} / y$ pairs of $\left(C_{1}, C_{2}\right)$ where the ciphertexts say $C_{3}$ and $C_{4}$ that $C_{1}$ and $C_{2}$ are related to are also in the same structure, i.e. $M^{2} \cdot 2^{2 t_{f}+2 r_{b}-2 n}$ possible quartets $\left(C_{1}, C_{2}, C_{3}, C_{4}\right)$ under $\left(K_{1}, K_{2}, K_{3}, K_{4}\right)$. Suppose that the corresponding plaintext is $\left(P_{1}, P_{2}, P_{3}, P_{4}\right)$. We check that both $P_{1} \oplus P_{2}$ and $P_{3} \oplus P_{4}$ are in $U_{b}$. The filtering process is achieved by creating a hash table of $2^{t_{b}}$ differences of $U_{b}$ and checking whether the differences of $P_{1} \oplus P_{2}$ and $P_{3} \oplus P_{4}$ are in the hash table. The probability that both $P_{1} \oplus P_{2}$ and $P_{3} \oplus P_{4}$ are in $U_{b}$ is $2^{\left(t_{b}-r_{b}\right) \times 2}$, so $M^{2} \cdot 2^{2 t_{f}+2 t_{b}-2 n}$ quartets will be left. In total this step takes $M^{2} \cdot 2^{2 t_{f}+2 r_{b}-2 n}\left(1+2^{t_{b}-r_{b}}\right)$ memory accesses.

Step 6 deduces the right subkey from the remaining quartets. Note that a right quartet satisfies $E_{b}^{K_{1}}\left(P_{1}\right) \oplus E_{b}^{K_{2}}\left(P_{2}\right)=E_{b}^{K_{3}}\left(P_{3}\right) \oplus E_{b}^{K_{4}}\left(P_{4}\right)=\alpha$ and key differences $\Delta_{1}, \Delta_{2}$ are chosen in advance and known. With these two key differences, $K_{2}, K_{3}, K_{4}$ can be computed from $K_{1}$. A right quartet must agree on the $m_{b}$ bits of $K_{1}$. There are $2^{t_{b}}$ possible input differences that may lead to $\alpha$ difference after $E_{b}$. Therefore, $2^{m_{b}-t_{b}}$ subkeys on average take one of the differences in $U_{b}$ to $\alpha$. For two pairs in a quartet, they agree on $\left(2^{m_{b}-t_{b}}\right)^{2} / 2^{m_{b}}=2^{m_{b}-2 t_{b}}$ subkeys for $E_{b}$. Do the same analysis for $E_{f}$ part with the corresponding ciphertexts, and we get $2^{m_{f}-2 t_{f}}$ subkey suggestions for $E_{f}$. Each remaining quartet suggests $2^{m_{b}+m_{f}-2 t_{b}-2 t_{f}}$ subkeys. There are $M^{2} \cdot 2^{2 t_{f}+2 t_{b}-2 n} \cdot 2^{m_{b}+m_{f}-2 t_{b}-2 t_{f}}=$ $M^{2} \cdot 2^{m_{b}+m_{f}-2 n}$ hits. For a wrong key, there are $M^{2} \cdot 2^{-2 n}(<1 / 16) h_{i t s}{ }^{5}$, while for a right key there are $s(e . g ., s=4)$ hits. Using hash tables, this step can be implemented with $M^{2} \cdot 2^{2 t_{f}+2 t_{b}-2 n} \cdot 2^{m_{b}-t_{b}} \cdot 2+M^{2} \cdot 2^{2 t_{f}+2 t_{b}-2 n} \cdot 2^{m_{f}-t_{f}} \cdot 2=M^{2} \cdot 2^{t_{b}+t_{f}-2 n+1}\left(2^{m_{b}+t_{f}}+2^{m_{f}+t_{b}}\right)$ memory accesses.

Step 7 requires a time complexity of $2^{k-h}$ encryptions.

Overall, the complexities of the rectangle attack are as follows.

- Data complexity: $D=4 M$ chosen plaintexts, where $M=\sqrt{s} \cdot 2^{n / 2} / \hat{p} \hat{q}$ and $s$ is the expected number of right quartets.

- Time complexity: $4 M+2 \cdot M^{2} \cdot 2^{r_{f}-n}+2 \cdot M^{2} \cdot 2^{t_{f}-n}+M^{2} \cdot 2^{2 t_{f}+2 r_{b}-2 n}\left(1+2^{t_{b}-r_{b}}\right)+$ $M^{2} \cdot 2^{t_{b}+t_{f}-2 n+1}\left(2^{m_{b}+t_{f}}+2^{m_{f}+t_{b}}\right)$ memory accesses and $2^{k-h}$ encryptions.

- Memory complexity: $4 M+2^{t_{b}}+2^{t_{f}}+2^{m_{b}+m_{f}}$.

Further Explanation of Data Complexity At first, we construct $y$ structures of $2^{r_{b}}$ plaintexts each, all of which are encrypted with $K_{1}, K_{2}, K_{3}$ and $K_{4}$ respectively. In each structure, there are roughly $2^{r_{b}} \cdot 2^{r_{b}}=2^{2 r_{b}}$ pairs of plaintexts keyed by $K_{1}$ and $K_{2}$ (or $K_{3}$ and $K_{4}$ ) that satisfy the input difference $\alpha^{\prime}$. After the $E_{b}$ encryption process, only $2^{2 r_{b}} / 2^{r_{b}}=2^{r_{b}}$ pairs that hold the difference of $\alpha$ will remain. Then there are $y 2^{r_{b}}$ pairs that satisfy the input difference of the rectangle distinguisher in total for $K_{1}$ and $K_{2}$ as well as for $K_{3}$ and $K_{4}$. Thus, the number of possible quartets is $y 2^{r_{b}} \cdot y 2^{r_{b}}=y^{2} 2^{2 r_{b}}$. The number of right quartets $s$ can then be deduced as $s=y^{2} 2^{2 r_{b}} \cdot 2^{-n} \hat{p}^{2} \hat{q}^{2}$ which means $y=\sqrt{s} \cdot 2^{n / 2-r_{b}} / \hat{p} \hat{q}$. Therefore, the data complexity would be $D=4 M=4 y 2^{r_{b}}=4 \sqrt{s} \cdot 2^{n / 2} / \hat{p} \hat{q}$.

Success Probability According to [Sel08], the success probability of differential analysis is

$$
P_{s}=\int_{\frac{\sqrt{s S_{N}}-\Phi^{-1}(1-2-h)}{\sqrt{S_{N}+1}}}^{\infty} \phi(x) d x,
$$

where $S_{N}$ is the signal-to-noise ratio and $S_{N}=\frac{2^{-n} \hat{p}^{2} \hat{q}^{2}}{2^{-2 n}}$ in rectangle attacks.

\footnotetext{
${ }^{5} D=4 M<2^{n}$, then $M^{2} \cdot 2^{-2 n}<2^{-4}$.
} 


\subsection{Related-Tweakey Rectangle Attacks}

This subsection takes SKINNY-64-128 as an example to illustrate our attacks and the attack works similarly for other versions so we just provide the results.

The following Fig. 9 depicts a dedicated attack of 22 rounds SKINNY-64-128 using the 15-round related-tweakey rectangle distinguisher and extending 3.5 rounds before and after the distinguisher.

In the attack, we choose the cell position and the value of the tweakey difference such that a distinguisher with the optimal probability can be obtained. As there is no whitening key added before the first round, when retrieving the subtweakey, we can attack one round for free. The subtweakey bits that affect the input difference $\alpha$ of the distinguisher are marked specially in each round and shown in Table 4.

Table 4: The round tweakey differences $\Delta t k_{i}$ used in the key recovery phase.

\begin{tabular}{cll}
\hline Round $i$ & Row $(1)$ & Row $(2)$ \\
\hline 2 & $\left(\begin{array}{llll}b & 0 & 0 & 0\end{array}\right)$ & $\left(\begin{array}{llll}0 & 0 & 0 & 0\end{array}\right)$ \\
4 & $\left(\begin{array}{llll}0 & 0 & a & 0\end{array}\right)$ & $\left(\begin{array}{llll}0 & 0 & 0 & 0\end{array}\right)$ \\
19 & $\left(\begin{array}{llll}0 & 0 & 0 & 0\end{array}\right)$ & $\left(\begin{array}{llll}0 & 0 & c & 0\end{array}\right)$ \\
21 & $\left(\begin{array}{lllll}0 & 0 & 0 & 0\end{array}\right)$ & $\left(\begin{array}{llll}0 & d & 0 & 0\end{array}\right)$ \\
\hline
\end{tabular}

Specifically, $r_{b}=\log _{2}\left(15^{4}\right)=15.6, t_{b}=\log _{2}\left(15^{3}\right)+\log _{2} 6=14.3, m_{b}=10 c=40$, $r_{f}=\log _{2}\left(15^{8}\right)=31.3, t_{f}=\log _{2}\left(15^{7}\right)+\log _{2} 8=30.3$ and $m_{f}=8 c=32$. As we have analyzed previously, the probability of the related-tweakey rectangle distinguisher is $\hat{p}^{2} \hat{q}^{2}=\left(2^{-3.9}\right)^{7} \cdot 2^{-2.4} \cdot\left(2^{-3.9}\right)^{6} \cdot 2^{-4}=2^{-57.1}$.

The total data complexity for 22-round SKINNY64-128 is $D=4 \cdot \sqrt{s} \cdot 2^{n / 2} / \hat{p} \hat{q}=$ $4 \cdot \sqrt{s} \cdot 2^{32} \cdot 2^{28.5}=\sqrt{s} \cdot 2^{62.5}$, i.e. if we choose $s=4$, the data complexity would be $2^{63.5}$. The time complexity required is $2^{110.9}$ memory access and $2^{108}$ encryptions, and the memory complexity is $2^{63.5}$. If we reduce the related-tweakey rectangle distinguisher by one round, i.e., a 14-round distinguisher, the data complexity and time complexity could be highly reduced. The probability of the 14-round distinguisher is $\hat{p}^{2} \hat{q}^{2}=\left(2^{-3.9}\right)^{3} \cdot\left(2^{-3.9}\right)^{6} \cdot 2^{-4}=$ $2^{-39.1}$. Using the same tweakey recovery procedure to extend the distinguisher 3.5 rounds at both sides of the distinguisher, the data complexity for 21-round attack is $D=4 \cdot \sqrt{s} \cdot 2^{n / 2} / \hat{p} \hat{q}=4 \cdot \sqrt{s} \cdot 2^{32} \cdot 2^{19.5}=\sqrt{s} \cdot 2^{53.5}=2^{54.5}$ (with $s=4$ ). The corresponding time complexity is $2^{93.9}$ memory access and $2^{88}$ encryptions, and the memory complexity is $2^{54.5}$.

Table 5 summarizes the results of related-tweakey rectangle attacks on all versions of SKINNY. It shows that the attack is more effective on TK2 and TK3 versions which is consistent with the fact that the attack takes advantage of the key schedule of SKINNY, e.g., 27 rounds of SKINNY-64-192 can be covered. Besides, we can see that the attack covers the same rounds whatever the block size is (64-bit or 128-bit).

\section{Related-Tweakey Differential Trails and Rectangle At- tacks}

In the previous sections, truncated differential trails are investigated and used to attack reduced versions of SKINNY. This section focuses on differential trails of SKINNY. In the specification of SKINNY [BJK ${ }^{+} 16 \mathrm{~b}$ ], the authors only gave lower bounds on the number of differential active Sboxes in SKINNY. It is not clear whether exact differential trails satisfying the lower bounds exist or not, especially for SKINNY-128 which employs a differentially non-optimal 8-bit Sbox. This section sheds some light on it and gives some good related-tweakey differential trails for both SKINNY-64 and SKINNY-128 on which boomerang distinguishers can also be constructed. 


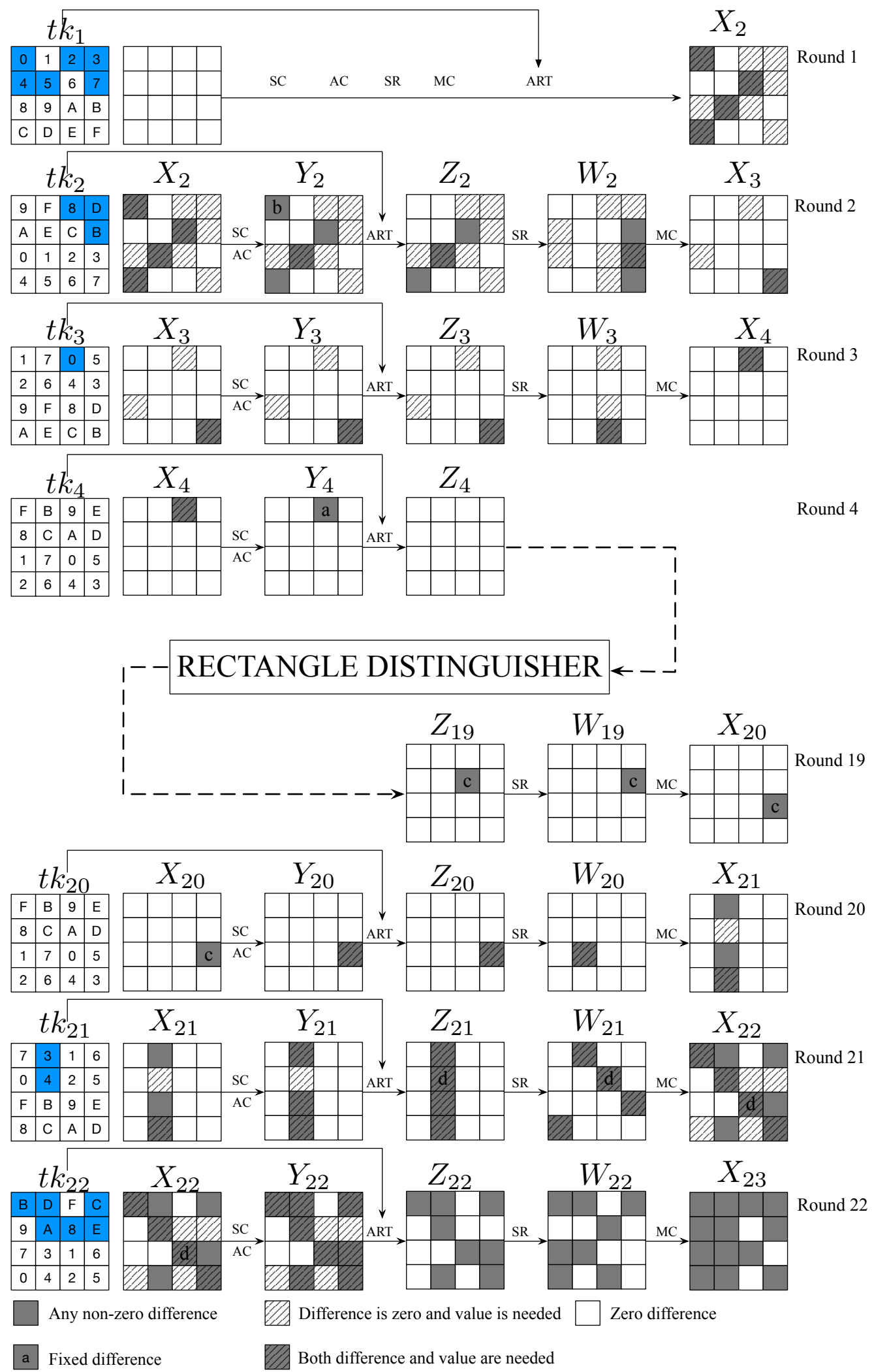

Figure 9: Tweakey rovery attack on 22-round SKINNY-64-128 
Table 5: Results of related-tweakey rectangle attack on SKINNY

\begin{tabular}{rrcccccccc}
\hline & & \#Rounds & $R_{d}$ & $R_{b}$ & $R_{f}$ & Data & Time & Memory & $P_{s}$ \\
\hline \multirow{2}{*}{ TK1 } & $64-64$ & 17 & 11 & 3.5 & 2.5 & $2^{54.5}$ & $2^{55.7}$ & $2^{54.5}$ & $97.72 \%$ \\
& $128-128$ & 17 & 11 & 3.5 & 2.5 & $2^{107}$ & $2^{113}$ & $2^{107}$ & $97.72 \%$ \\
\hline \multirow{2}{*}{ TK2 } & $64-128$ & 21 & 14 & 3.5 & 3.5 & $2^{54.5}$ & $2^{93.9}$ & $2^{54.5}$ & $97.72 \%$ \\
& $64-128$ & 22 & 15 & 3.5 & 3.5 & $2^{63.5}$ & $2^{110.9}$ & $2^{63.5}$ & $94.03 \%$ \\
& $128-256$ & 22 & 15 & 3.5 & 3.5 & $2^{127}$ & $2^{235.6}$ & $2^{127}$ & $95.37 \%$ \\
\hline \multirow{2}{*}{ TK3 } & $64-192$ & 27 & 19 & 4.5 & 3.5 & $2^{63.5}$ & $2^{156.5}$ & $2^{80}$ & $91.56 \%$ \\
& $128-384$ & 27 & 19 & 4.5 & 3.5 & $2^{127}$ & $2^{351}$ & $2^{160}$ & $93.11 \%$ \\
\hline
\end{tabular}

\subsection{Strategies for Finding Differential Trails}

In this section our aim is to find optimal differential trails for SKINNY-64 and good differential trails for SKINNY-128 in a reasonable time. It is challenging to directly find optimal differential trails, even for block ciphers using 4-bit Sboxes, so we propose an indirect method for finding optimal differential trails for SKINNY-64, which is described as follows.

1. For an $r$-round SKINNY-64, find all truncated differential trails with $A S=A S_{\text {min }}$ active Sboxes, where $A S_{\text {min }}$ denotes the minimal number of active Sboxes of truncated differential trails;

2. Based on the truncated differential trails, search for a best differential trail, namely the trail with the highest probability. If the best differential trail obtained has probability $p=2^{-2 A S_{\min }-i}, i \in\{0,1,2\}$, then this trail must be the optimal trail for an $r$-round SKINNY-64; otherwise, go to Step 3;

3. For $A S=A S_{\min }+1$ to $\left\lfloor-\log _{2} p / 2\right\rfloor$, find all truncated differential trails with $A S$ active Sboxes. Based on the truncated differentials, search for a best differential trail with probability $p^{\prime}$. If $p>p^{\prime}$, let $p=p^{\prime}$. Until $p=2^{-2 A S-i}, i \in\{0,1,2\}$ is satisfied, then the trail related to $p$ is the optimal trail for an $r$-round SKINNY.

Following the designers of SKINNY $\left[\mathrm{BJK}^{+} 16 \mathrm{~b}\right]$, we generate a Mixed-Integer Linear Programming (MILP) model to get truncated differential trails. Basic ideas of converting a differential searching problem into inequalities over integers are introduced in [MWGP11, $\left.\mathrm{SHS}^{+} 13, \mathrm{SHW}^{+} 14\right]$. Once the active pattern, i.e., a truncated differential trail is given, the search for finding the optimal trail with MILP solvers is greatly sped up. In this way optimal differential trails can be found for SKINNY-64 as long as the number of truncated differential trails that needs to be traversed is reasonable, say less than 5000 .

However, for SKINNY-128, 8-bit Sboxes are too heavy for Mixed-Integer Linear Programming solvers, so a dedicated searching algorithm is used after obtaining a truncated differential trail with the minimal number of active Sboxes. Our idea of our algorithm is that given a truncated differential trail, there exists a few free active master tweakey bytes for which we could traverse all their nonzero values. Once the master tweakey difference is fixed, the round tweakey differences can be calculated since the key schedule is linear. With the round tweakey differences known, it is easy to find differential trails or verify there is no differential trails following the active pattern. In the search for differential trails of SKINNY-128, only a few truncated differential of minimal number of active Sboxes are considered. 


\subsection{Results of Differential Trails}

Table 6 lists the results of SKINNY-64 from 6 rounds to 13 rounds under related-tweakey model. For each of TK1, TK2 and TK3, the first line shows the number of minimal active Sboxes; the second line presents the probability $p_{1}$ of best trails following the truncated differentials with the minimal number active Sboxes; and the last line shows the probability $p_{2}$ of optimal trails ${ }^{6}$. Under TK1, differential trails with probability $2^{-2 A S_{\text {min }}}$ are found in all cases. However, under TK2, and TK3, as the number of rounds increases, the probability of the optimal trail is much lower than $2^{-2 A S_{\min }}$.

Table 6: Bounds on the number of active Sboxes and probability of trails in SKINNY-64 under related-tweakey models

\begin{tabular}{cc|cccccccc}
\hline \multicolumn{2}{c|}{ \#rounds } & 6 & 7 & 8 & 9 & 10 & 11 & 12 & 13 \\
\hline TK1 & $A S_{\min }$ & 6 & 10 & 13 & 16 & 23 & 32 & 38 & 41 \\
& $-\log _{2} p$ & 12 & 20 & 26 & 32 & 46 & 64 & 76 & 82 \\
\hline TK2 & $A S_{\min }$ & 2 & 3 & 6 & 9 & 12 & 16 & 21 & 25 \\
& $-\log _{2} p_{1}$ & 4 & 6 & 12 & 20 & None† & 35 & 49 & 55 \\
& $-\log _{2} p_{2}$ & 4 & 6 & 12 & 20 & 28 & 35 & 48 & 55 \\
\hline TK3 & $A S_{\min }$ & 0 & 1 & 2 & 3 & 6 & 10 & 13 & 16 \\
& $-\log _{2} p_{1}$ & 0 & 2 & 4 & 6 & 12 & 20 & 28 & 43 \\
& $-\log _{2} p_{2}$ & 0 & 2 & 4 & 6 & 12 & 20 & 28 & 38 \\
\hline
\end{tabular}

$\dagger^{\dagger}$ No solution is found for all truncated differentials with the minimal number of active Sboxes.

For SKINNY-128, only the cases that are promising in rectangle attacks are considered and the results are shown in Table 7. Note that the highest probability for the 8-bit Sbox used in SKINNY-128 is also $2^{-2}$. As can be seen from the results that to make the total number of active Sboxes lower, the average probability of each Sbox is also much lower. For example, the minimal number of active Sboxes of 9-round SKINNY-128-256 is 9 , and following one of the truncated differential that satisfies this bound, the best trail has probability $2^{-34.42}$. While extending an 8-round trail with 6 active Sboxes one round back, we get a 9 -round trail with 10 active Sboxes and probability $2^{-20}$.

Table 7: Bounds on the number of active Sboxes and probability of trails in SKINNY-128 under related-tweakey models

\begin{tabular}{|c|c|c|c|c|c|c|c|c|}
\hline \multicolumn{2}{|c|}{ \#rounds } & 6 & 7 & 8 & 9 & 10 & 11 & 12 \\
\hline \multirow[t]{2}{*}{ TK1 } & $A S_{\min }$ & 6 & 10 & 13 & & & & \\
\hline & $-\log _{2} p$ & 12 & 20 & $54(36) \ddagger$ & & & & \\
\hline \multirow[t]{2}{*}{ TK2 } & $A S_{\min }$ & & & 6 & 9 & & & \\
\hline & $-\log _{2} p$ & & & 12 & $34.42(20)$ & & & \\
\hline \multirow[t]{2}{*}{ TK3 } & $A S_{\min }$ & & & & & 6 & 10 & 13 \\
\hline & $-\log _{2} p$ & & & & & 12 & 21 & $62.83(37)$ \\
\hline
\end{tabular}

$\ddagger$ The values in parentheses are the probability of $r$-round differential trails obtained by extending a $(r-1)$-round differential trail.

These results show a trend that it is not likely to reach the bounds of $2^{-2 A S_{\min }}$ as the number of rounds increase for both SKINNY-64 and SKINNY-128.

\footnotetext{
${ }^{6}$ For TK1, the second line and the third line are identical, so only one line is kept.
} 


\subsection{Related-Tweakey Rectangle Attacks}

Based on differential trails in the previous subsection, rectangle distinguishers can be constructed. We follow the notations in Section 4. Suppose $E^{\prime}=E_{1} \circ E_{0}$ is the rectangle distinguisher. The probability of the upper trail for $E_{0}$ under tweakey difference $\Delta_{1}$ (resp. lower trail for $E_{1}$ under $\left.\Delta_{2}\right) \operatorname{Pr}\left(\alpha \stackrel{\Delta_{1}}{\longrightarrow} \beta\right)\left(\operatorname{resp} . \operatorname{Pr}\left(\gamma \stackrel{\Delta_{2}}{\longrightarrow} \delta\right)\right)$ is denoted by $p$ (resp. $q)$. If multiple trails are considered for $E_{0}$ or $E_{1}$, we denote $\sqrt{\Sigma_{i} \operatorname{Pr}^{2}\left(\alpha \stackrel{\Delta_{1}}{\longrightarrow} \beta_{i}\right)}$ (resp. $\sqrt{\Sigma_{j} \operatorname{Pr}^{2}\left(\gamma_{j} \stackrel{\Delta_{2}}{\longrightarrow} \delta\right)}$ ) by $\hat{p}$ (resp. $\hat{q}$ ). In the tweakey recovery attack, the same notations of $E=E_{f} \circ E_{1} \circ E_{0} \circ E_{b}, m_{b}, r_{b}, t_{b}, m_{f}, r_{f}$ and $t_{f}$ are used, and the number of right quartets $s$ is set to be 4 and the success probability of attacks in this section is greater than $95 \%$. Under TK1 where the key size is as large as the block size, the rectangle attack does not work as well as under TK2 and TK3. Thus, in this section only distinguishers under TK2 and TK3 are presented, together with the tweakey recovery attacks using the adapted key recovery algorithm in Section 4.3.

\subsubsection{1-Round attack on SKINNY-64-128}

We construct a 17-round rectangle distinguisher by combining an 8-round upper trail and a 9-round lower trail. Details of these two trails are shown in Table 12 . For $E_{0}$, if we fix the input difference and the tweakey difference according to the 8-round trail, there are 5477 trails which belong to 1563 differentials, and $\hat{p}=2^{-7.15}$. Similarly for $E_{1}$, if we fix the output difference and the tweakey difference according to the 9-round trail, there are 24 trails which belong to 6 differentials, and $\hat{q}=2^{-17.21}$. By extending 3 rounds backward and one round forward, we get following parameters: $r_{b}=13 c, t_{b}=8 c, m_{b}=10 c$, $r_{f}=12 c, t_{f}=7 c, m_{f}=4 c$ where $c=4$ is the cell size. Using the adapted key recovery algorithm, a 21-round version of SKINNY-64-128 can be attacked with data complexity of $D=2^{59.36}$ chosen plaintexts, time complexity of $2^{115.72}$ memory accesses and $2^{108}$ encryptions, and memory complexity of $2^{59.36}$ blocks. The probability of success is $97.59 \%$.

\subsubsection{2-Round attack on SKINNY-128-256}

Following the truncated differentials with the minimal number of active Sboxes, we found an 8-round trail of probability $2^{-12}$ and a 9 -round trail of probability $2^{-34.42}$. By extending the 8-round trail one round backward, we get a 9-round trail with probability $2^{-20}$ which is higher than the probability of the 9-round trail obtained directly from a 9 -round truncated differential with the minimal number of active Sboxes. These two 9 -round trails are displayed in Table 13 . We choose the 9-round trail with probability $2^{-34.42}$ for $E_{0}$ and the other one with probability $2^{-20}$ for $E_{1}$. If the output difference and the tweakey difference are fixed, there are $2^{5}$ trails with the same probability for $E_{1}$, so $\hat{q}=2^{-17.5}$ and $\hat{p} \hat{q}=p \hat{q}=2^{-51.92}$ for the 18 -round rectangle distinguisher. Using the distinguisher from Round 4 to Round 21, we can attack 22 rounds with following parameters: $r_{b}=14 c, t_{b}=8 c, m_{b}=10 c, r_{f}=12 c, t_{f}=8 c, m_{f}=5 c$ where $c=8$ is the cell size. Figure 10 gives a visualized view of the key recovery attack. Using the adapted key recovery algorithm, a 22-round version of SKINNY-128-256 can be attacked with data complexity of $D=2^{118.92}$ chosen plaintexts, time complexity of $2^{250.84}$ memory accesses and $2^{248}$ encryptions, and memory complexity of $2^{120}$ blocks.

\subsubsection{6-Round attack on SKINNY-64-192}

The best 11-round related-tweakey differential trail for SKINNY-64-192 has probability $2^{-20}$ as shown in Table 14 . The same trail is used for both $E_{0}$ and $E_{1}$ to get a 22-round distinguisher. If we fix input difference (output difference) and the tweakey difference, there are many trails. Taking 5000 trails into consideration, $\hat{p}=2^{-14.51}, \hat{q}=2^{-12.96}$. 


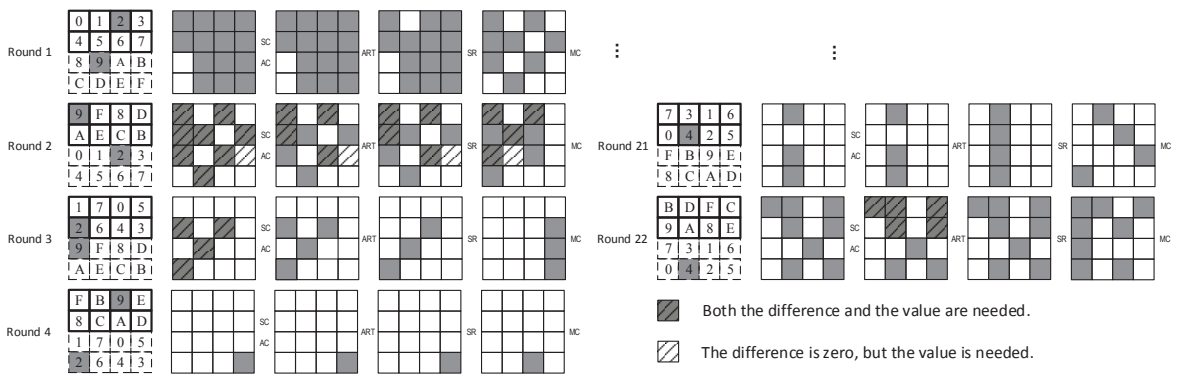

Figure 10: Tweakey recovery attack of 22-round SKINNY-128-256 using a 18-round related-tweakey rectangle distinguisher from Round 4 to Round 21

By extending 2 rounds backward and 2 rounds forward, we get following parameters: $r_{b}=13 c, t_{b}=8 c, m_{b}=6 c, r_{f}=16 c, t_{f}=13 c, m_{f}=12 c$ where $c=4$. Consequently, 26 rounds of SKINNY-64-192 can be attacked with data complexity of $D=2^{62.47}$ chosen plaintexts, time complexity of $2^{160.94}$ memory accesses and $2^{154}$ encryptions, and memory complexity of $2^{72}$ blocks.

\subsubsection{7-Round attack on SKINNY-128-384}

The 11-round related-tweakey differential trail for SKINNY-128-384 we found has probability $2^{-21}$, and with the same input difference and output difference there are two trails of the same probability. By extending this 11-round differential trail backward for one round we get a 12 -round trail with probability $2^{-37}$. We connect the 11 -round trail and the 12-round trail to get a 23-round rectangle distinguisher. Using the boomerang switching technique [BK09] at the meeting point of two trails, four Sboxes of the lower trail can be saved. If we fix the tweakey difference and the output difference, there are $2^{8}$ trails with the same probability, thus $\hat{q}=2^{-25}$. Since $\hat{p}=2^{-21}, \hat{p} \hat{q}=2^{-45}$. By extending 2 rounds backward and 2 rounds forward, we get following parameters: $r_{b}=13 c, t_{b}=8 c, m_{b}=6 c$, $r_{f}=16 c, t_{f}=13 c, m_{f}=12 c$ where $c=8$. Thus, a 27-round SKINNY-128-384 can be attacked with data complexity of $D=2^{112}$ chosen plaintexts, time complexity of $2^{300}$ memory accesses and $2^{331}$ encryptions, and memory complexity of $2^{144}$ blocks.

Experimental verification of the distinguishers. In order to verify that no contradiction happens at the meeting point of the two trails of our distinguishers, experiments are mounted to search for right quartets where the distinguishers are reduced to two middle rounds, i.e. the last round of the upper trail and the first round of the lower trail. In this way, right quartets can be found practically as long as there exists no contradiction at the meeting point. As a result, right quartets are obtained for all four distinguishers in this section, and the time complexity for finding a right quartet is much lower than expected. More details of the experiments are shown in Table 11.

Results of related-tweakey rectangle attacks on SKINNY- $n-2 n$ and SKINNY- $n-3 n$ are summarized in Table 8. As can be seen that even though differential trails based rectangle distinguishers cover more rounds compared with rectangle distinguishers based on truncated differential trails as in Table 5, the total number of rounds attacked is not necessarily more.

\section{Conclusion}

This paper analyzes the security of SKINNY under related-tweakey settings using impossible differential and rectangle attacks which have a data complexity below codebook. The 
Table 8: Results of related-tweakey rectangle attacks on SKINNY- $n-2 n$ and SKINNY- $n-3 n$

\begin{tabular}{lrcccccccc}
\hline Model & Version & \#Rounds & $R_{d}$ & $R_{b}$ & $R_{f}$ & Data & Time & Memory & $P_{s}$ \\
\hline TK2 & $64-128$ & 21 & 17 & 3 & 1 & $2^{59.36}$ & $2^{115.72}$ & $2^{59.36}$ & $97.59 \%$ \\
& $128-256$ & 22 & 18 & 3 & 1 & $2^{118.92}$ & $2^{251.03}$ & $2^{120}$ & $97.72 \%$ \\
\hline TK3 & $64-192$ & 26 & 22 & 2 & 2 & $2^{62.47}$ & $2^{160.95}$ & $2^{72}$ & $95.56 \%$ \\
& $128-384$ & 27 & 23 & 2 & 2 & $2^{112}$ & $2^{331}$ & $2^{144}$ & $97.72 \%$ \\
\hline
\end{tabular}

results show that 19,23 and 27 rounds of SKINNY-n-n/2n/3n can be attacked respectively with truncated differential trails. This paper also analyzes the security of SKINNY using actual differential trails and presents results for the rectangle attacks under TK2 and TK3. For SKINNY-128, better attack complexities are obtained (in terms of data and memory) compared to truncated differential trails. For SKINNY-64, the results show a trend that as the number of rounds increases, the probability of optimal differential trails is much lower than the probability derived from lower bounds of active Sboxes in SKINNY. Another interesting outcome of this work shows that the increased number of rounds in the distinguisher may not necessarily lead to more rounds attacked.

\section{Acknowledgements}

The authors would like to thank the anonymous referees for their helpful comments. The first author is supported by the National Key Research and Development Program of China under grant 2017YFB0802704. The third author is supported by the National Key Basic Research Program of China (2013CB834203) and the National Natural Science Foundation of China (Grants 61472417, 61472415, 61402469, and 61672516).

\section{References}

$\left[\mathrm{ABC}^{+} 17\right]$ Ralph Ankele, Subhadeep Banik, Avik Chakraborti, Eik List, Florian Mendel, Siang Meng Sim, and Gaoli Wang. Related-key impossible-differential attack on reduced-round skinny. In Dieter Gollmann, Atsuko Miyaji, and Hiroaki Kikuchi, editors, Applied Cryptography and Network Security - 15th International Conference, ACNS 2017, Kanazawa, Japan, July 10-12, 2017, Proceedings, volume 10355 of Lecture Notes in Computer Science, pages 208228. Springer, 2017.

[AHMN13] Jean-Philippe Aumasson, Luca Henzen, Willi Meier, and María Naya-Plasencia. Quark: A lightweight hash. J. Cryptology, 26(2):313-339, 2013.

[BBS05] Eli Biham, Alex Biryukov, and Adi Shamir. Cryptanalysis of skipjack reduced to 31 rounds using impossible differentials. J. Cryptology, 18(4):291-311, 2005.

[BD08] Steve Babbage and Matthew Dodd. The MICKEY stream ciphers. In Matthew J. B. Robshaw and Olivier Billet, editors, New Stream Cipher Designs - The eSTREAM Finalists, volume 4986 of Lecture Notes in Computer Science, pages 191-209. Springer, 2008.

[BDK01] Eli Biham, Orr Dunkelman, and Nathan Keller. The rectangle attack rectangling the serpent. In Birgit Pfitzmann, editor, Advances in Cryptology EUROCRYPT 2001, International Conference on the Theory and Application of Cryptographic Techniques, Innsbruck, Austria, May 6-10, 2001, Proceeding, 
volume 2045 of Lecture Notes in Computer Science, pages 340-357. Springer, 2001.

[BDK02] Eli Biham, Orr Dunkelman, and Nathan Keller. New results on boomerang and rectangle attacks. In Joan Daemen and Vincent Rijmen, editors, Fast Software Encryption, 9th International Workshop, FSE 2002, Leuven, Belgium, February 4-6, 2002, Revised Papers, volume 2365 of Lecture Notes in Computer Science, pages 1-16. Springer, 2002.

[BJK $\left.{ }^{+} 16 \mathrm{a}\right]$ Christof Beierle, Jérémy Jean, Stefan Kölbl, Gregor Leander, Amir Moradi, Thomas Peyrin, Yu Sasaki, Pascal Sasdrich, and Siang Meng Sim. https: //sites.google.com/site/skinnycipher/cryptanalysis-competition, 2016.

[BJK $\left.{ }^{+} 16 \mathrm{~b}\right]$ Christof Beierle, Jérémy Jean, Stefan Kölbl, Gregor Leander, Amir Moradi, Thomas Peyrin, Yu Sasaki, Pascal Sasdrich, and Siang Meng Sim. The SKINNY family of block ciphers and its low-latency variant MANTIS. In Matthew Robshaw and Jonathan Katz, editors, Advances in Cryptology CRYPTO 2016 - 36th Annual International Cryptology Conference, Santa Barbara, CA, USA, August 14-18, 2016, Proceedings, Part II, volume 9815 of Lecture Notes in Computer Science, pages 123-153. Springer, 2016.

[BJK $\left.{ }^{+} 16 c\right]$ Christof Beierle, Jérémy Jean, Stefan Kölbl, Gregor Leander, Amir Moradi, Thomas Peyrin, Yu Sasaki, Pascal Sasdrich, and Siang Meng Sim. The SKINNY family of block ciphers and its low-latency variant MANTIS. IACR Cryptology ePrint Archive, 2016:660, 2016.

[BK09] Alex Biryukov and Dmitry Khovratovich. Related-key cryptanalysis of the full AES-192 and AES-256. In Mitsuru Matsui, editor, Advances in Cryptology - ASIACRYPT 2009, 15th International Conference on the Theory and Application of Cryptology and Information Security, Tokyo, Japan, December 6-10, 2009. Proceedings, volume 5912 of Lecture Notes in Computer Science, pages 1-18. Springer, 2009.

$\left[\mathrm{BKL}^{+} 07\right]$ Andrey Bogdanov, Lars R. Knudsen, Gregor Leander, Christof Paar, Axel Poschmann, Matthew J. B. Robshaw, Yannick Seurin, and C. Vikkelsoe. PRESENT: an ultra-lightweight block cipher. In Pascal Paillier and Ingrid Verbauwhede, editors, Cryptographic Hardware and Embedded Systems - CHES 2007, 9th International Workshop, Vienna, Austria, September 10-13, 2007, Proceedings, volume 4727 of Lecture Notes in Computer Science, pages 450-466. Springer, 2007.

$\left[\mathrm{BKL}^{+} 11\right]$ Andrey Bogdanov, Miroslav Knezevic, Gregor Leander, Deniz Toz, Kerem Varici, and Ingrid Verbauwhede. spongent: A lightweight hash function. In Preneel and Takagi [PT11], pages 312-325.

[BNS14] Christina Boura, María Naya-Plasencia, and Valentin Suder. Scrutinizing and improving impossible differential attacks: Applications to clefia, camellia, lblock and simon. In Sarkar and Iwata [SI14], pages 179-199.

[BSS $\left.{ }^{+} 13\right]$ Ray Beaulieu, Douglas Shors, Jason Smith, Stefan Treatman-Clark, Bryan Weeks, and Louis Wingers. The SIMON and SPECK families of lightweight block ciphers. IACR Cryptology ePrint Archive, 2013:404, 2013.

[CP08] Christophe De Cannière and Bart Preneel. Trivium. In Matthew J. B. Robshaw and Olivier Billet, editors, New Stream Cipher Designs - The eSTREAM 
Finalists, volume 4986 of Lecture Notes in Computer Science, pages 244-266. Springer, 2008.

[GPP11] Jian Guo, Thomas Peyrin, and Axel Poschmann. The PHOTON family of lightweight hash functions. In Phillip Rogaway, editor, Advances in Cryptology - CRYPTO 2011 - 31st Annual Cryptology Conference, Santa Barbara, CA, USA, August 14-18, 2011. Proceedings, volume 6841 of Lecture Notes in Computer Science, pages 222-239. Springer, 2011.

[GPPR11] Jian Guo, Thomas Peyrin, Axel Poschmann, and Matthew J. B. Robshaw. The LED block cipher. In Preneel and Takagi [PT11], pages 326-341.

[HJMM08] Martin Hell, Thomas Johansson, Alexander Maximov, and Willi Meier. The grain family of stream ciphers. In Matthew J. B. Robshaw and Olivier Billet, editors, New Stream Cipher Designs - The eSTREAM Finalists, volume 4986 of Lecture Notes in Computer Science, pages 179-190. Springer, 2008.

[JNP14] Jérémy Jean, Ivica Nikolic, and Thomas Peyrin. Tweaks and keys for block ciphers: The TWEAKEY framework. In Palash Sarkar and Tetsu Iwata, editors, Advances in Cryptology - ASIACRYPT 2014 - 20th International Conference on the Theory and Application of Cryptology and Information Security, Kaoshiung, Taiwan, R.O.C., December 7-11, 2014, Proceedings, Part II, volume 8874 of Lecture Notes in Computer Science, pages 274-288. Springer, 2014.

[KKS00] John Kelsey, Tadayoshi Kohno, and Bruce Schneier. Amplified boomerang attacks against reduced-round MARS and serpent. In Bruce Schneier, editor, Fast Software Encryption, 7th International Workshop, FSE 2000, New York, NY, USA, April 10-12, 2000, Proceedings, volume 1978 of Lecture Notes in Computer Science, pages 75-93. Springer, 2000.

[Knu98] Lars Knudsen. Deal - a 128-bit block cipher. In NIST AES Proposal, 1998.

[MWGP11] Nicky Mouha, Qingju Wang, Dawu Gu, and Bart Preneel. Differential and linear cryptanalysis using mixed-integer linear programming. In Chuankun Wu, Moti Yung, and Dongdai Lin, editors, Information Security and Cryptology 7th International Conference, Inscrypt 2011, Beijing, China, November 30 December 3, 2011. Revised Selected Papers, volume 7537 of Lecture Notes in Computer Science, pages 57-76. Springer, 2011.

[PT11] Bart Preneel and Tsuyoshi Takagi, editors. Cryptographic Hardware and Embedded Systems - CHES 2011 - 13th International Workshop, Nara, Japan, September 28 - October 1, 2011. Proceedings, volume 6917 of Lecture Notes in Computer Science. Springer, 2011.

[Sel08] Ali Aydın Selçuk. On probability of success in linear and differential cryptanalysis. Journal of Cryptology, 21(1):131-147, 2008.

$\left[\mathrm{SHS}^{+} 13\right]$ Siwei Sun, Lei Hu, Ling Song, Yonghong Xie, and Peng Wang. Automatic security evaluation of block ciphers with s-bp structures against related-key differential attacks. In Dongdai Lin, Shouhuai Xu, and Moti Yung, editors, Information Security and Cryptology - 9th International Conference, Inscrypt 2013, Guangzhou, China, November 27-30, 2013, Revised Selected Papers, volume 8567 of Lecture Notes in Computer Science, pages 39-51. Springer, 2013. 
$\left[\mathrm{SHW}^{+} 14\right]$ Siwei Sun, Lei Hu, Peng Wang, Kexin Qiao, Xiaoshuang Ma, and Ling Song. Automatic security evaluation and (related-key) differential characteristic search: Application to simon, present, lblock, DES(L) and other bit-oriented block ciphers. In Sarkar and Iwata [SI14], pages 158-178.

[SI14] Palash Sarkar and Tetsu Iwata, editors. Advances in Cryptology - ASIACRYPT 2014 - 20th International Conference on the Theory and Application of Cryptology and Information Security, Kaoshiung, Taiwan, R.O.C., December 7-11, 2014. Proceedings, Part I, volume 8873 of Lecture Notes in Computer Science. Springer, 2014.

[SMB16] Sadegh Sadeghi, Tahere Mohammadi, and Nasour Bagheri. Cryptanalysis of Reduced round SKINNY Block Cipher. IACR Cryptology ePrint Archive, 2016:1120, 2016 .

[TAY16] Mohamed Tolba, Ahmed Abdelkhalek, and Amr M. Youssef. Impossible Differential Cryptanalysis of Reduced-Round SKINNY. IACR Cryptology ePrint Archive, 2016:1115, 2016.

[Wag99] David Wagner. The boomerang attack. In Lars R. Knudsen, editor, Fast Software Encryption, 6th International Workshop, FSE '99, Rome, Italy, March 24-26, 1999, Proceedings, volume 1636 of Lecture Notes in Computer Science, pages 156-170. Springer, 1999. 


\section{A Truncated Impossible Differentials}

Table 9 lists all the truncated impossible differentials we found under both single-tweakey model and related-tweakey models. These truncated impossible differentials are found by reusing the Mixed Integer Linear Programming model as in Section 5.

Table 9: Truncated impossible differentials

\begin{tabular}{|c|l|}
\hline Model & (active cell position in input, active cell position in output) \\
\hline SK & $(12,8),(13,8),(12,11),(14,9),(14,11),(15,10)$ \\
11 rounds & $(12,9),(13,9),(13,10),(15,8),(14,10),(15,11)$ \\
\hline \hline Model & $($ active cell position in key, active cell position in output $)$ \\
\hline TK1 & $(8,8),(9,10),(13,8),(10,9),(12,10),(13,11)$ \\
12 rounds & $(\mathbf{9}, \mathbf{9}),(10,8),(13,9),(14,9),(14,10),(15,10)$ \\
\hline $\begin{array}{c}\text { TK2 } \\
14 \text { rounds }\end{array}$ & $(8,8),(12,9),(14,8),(\mathbf{1 0}, \mathbf{1 0}),(12,10),(9,8)$ \\
\hline TK3 & $(9,8),(14,9),(15,9),(11,10),(15,10),(14,11)$ \\
16 rounds & $(9,9),(10,9),(11,10),(13,10),(11,9),(12,11)$ \\
\hline \hline Model & active cell position in key \\
\hline $\begin{array}{c}\text { TK1 } \\
12 \text { rounds }\end{array}$ & \\
\hline $\begin{array}{c}\text { TK2 } \\
14 \text { rounds }\end{array}$ & $8,9,10,11,12,13,14,15$ \\
\hline $\begin{array}{l}\text { TK3 } \\
16 \text { rounds }\end{array}$ & \\
\hline
\end{tabular}

Note that, in the specification of SKINNY $\left[\mathrm{BJK}^{+} 16 \mathrm{c}\right]$, the authors state that there are 16 such truncated impossible differentials under single-tweakey model where only one cell is active in both the input and output, while we could only find 12.

For related-tweakey models, no result is reported in $\left[\mathrm{BJK}^{+} 16 \mathrm{c}\right]$. Our impossible differentials under related-tweakey model start with a fully passive state and a master key with only a special active cell which makes the first $2 z-1$ subtweakeys have no active cells and end with one active cell after the SubCells operation of Round $r$ where $r$ is the number of rounds in the distinguisher. Therefore, in Table 9 each truncated impossible differential is represented with a tuple. As can be seen, there are 12 such impossible differentials under TK1/TK2/TK3 with 12/14/16 rounds. Table 9 also lists all the impossible differentials that start as well as end with a fully passive state.

\section{B Related Tweakey Impossible Differential Attack for SKINNY- $n-n$ and SKINNY-n-3n}

In this section, we present the details of our 19-round attack on SKINNY- $n-n$ using related tweakey impossible differential attack. After that we just list out the parameters and results of SKINNY- $n-3 n$.

\section{B.1 SKINNY-n-n}

Our 12-round distinguisher is placed between Round 5 to Round 17. A 6.5-round relatedtweakey differential in the forward direction (having prob. 1) starting at $Y_{5}$ (after the $S C$ and $A C$ operations in Round 5) is concatenated to a 5.5-round related-tweakey differential (having prob. 1) starting in the reverse direction from $Y_{17}$ (before the $A R T$ 

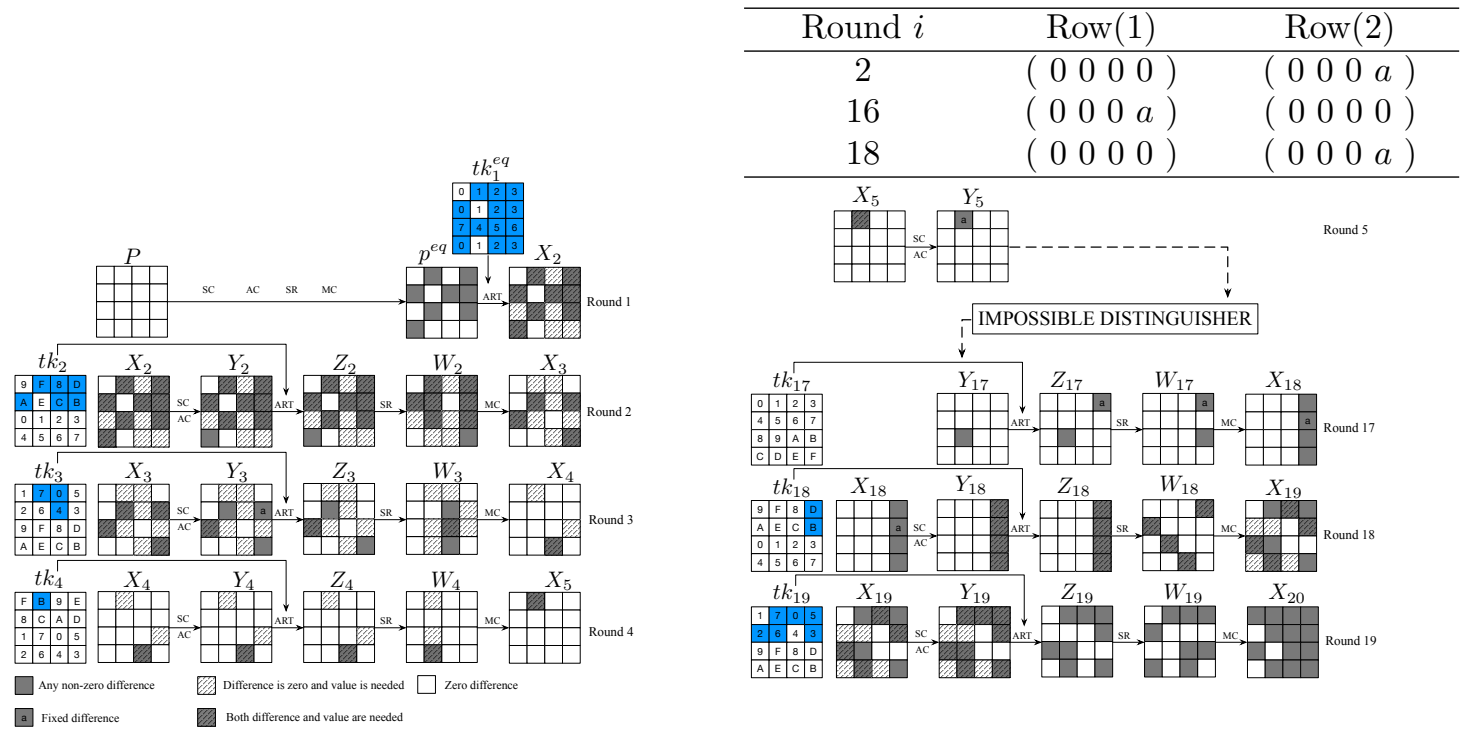

Figure 11: 19-round attack against SKINNY- $n-n$.

operation in Round 17). The contradiction happens in Round 12 at $X_{12}$ [12]. The 12-round related-tweakey impossible differential is:

$$
(0 a 00|0000| 0000 \mid 0000) \stackrel{12 y}{\longrightarrow}(0000|0000| 0 N 00 \mid 0000)
$$

where $a$ denotes a fixed non-zero difference and $N$ denotes any non-zero difference. This 12-round distinguisher is extended 4.5 rounds at the top and 2.5 rounds in the bottom to cover 7 rounds in the tweakey recovery phase as shown in Fig. 11. Following the notations explained in Section 3.2, here $E^{\prime}$ covers 12 rounds, $E_{f}\left(E_{b}\right)$ covers 2.5 (4.5) rounds, and $c_{\text {in }}=\left|\Delta_{\text {in }}\right|=8 c, c_{\text {out }}=7 c,\left|\Delta_{\text {out }}\right|=8 c$ and $\left|k_{\text {in }} \cup k_{\text {out }}\right|=14 c$ where $c$ is the cell size. The steps of our tweakey recovery phase are as follows:

Data collection Consider a pair of structures $S_{1}$ and $S_{2}$, where, each structure consists of $2^{\left|\Delta_{i n}\right|}=2^{8 c}$ plaintexts and for each plaintext pair $P_{1} \in S_{1} \& P_{2} \in S_{2}, P_{1} \oplus P_{2}=$

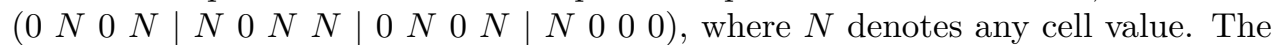
total number of possible plaintext pairs is $2^{16 c}$. Invert back the plaintexts in $S_{1}$ and $S_{2}$ by one keyless round to get the original plaintexts. Encrypt the pool $S_{1}$ under $(T K-1)^{1}$ and the pool $S_{2}$ under $(T K-1)^{2}$ to obtain the corresponding ciphertexts. For each ciphertext pair, check whether $n-\left|\Delta_{\text {out }}\right|=8 c$ bits are zero or not, i.e., $\Delta W_{19}[1,2,3,4,10,11,12,14]$ should be zero. Generate $2^{x}$ such pair of structures and repeat this for each pair of structures. In total there are $2^{x+2\left|\Delta_{i n}\right|}=2^{x+16 c}$ plaintext pairs. This step requires $2^{x+\left|\Delta_{i n}\right|+1}=2^{x+8 c+1}$ encryptions. Out of the total $2^{x+16 c}$ ciphertext pairs, $M=2^{x+2\left|\Delta_{i n}\right|-n+\left|\Delta_{\text {out }}\right|}=2^{x+8 c}$ pairs are expected to remain. With the help of a hash table, this step takes a memory complexity of $2^{\left|\Delta_{i n}\right|}=2^{8 c}{ }^{7}$

Tweakey recovery For $M$ pairs:

\footnotetext{
${ }^{7}$ For each pair of structures under $(T K-1)^{1}$ and $(T K-1)^{2}$, insert the messages of one structure into a hash table according to the the value of $W_{19}[1,2,3,4,10,11,12,14]$, and then use messages in the other structure to find message pairs under the related tweakeys such that $W_{19}[1,2,3,4,10,11,12,14]$ are equal by searching the built hash table. An attacker can do this for each structure pair. In this way, the time complexity is equal to the data complexity, and the memory complexity remains to be the size of one structure.
} 
(a) Check whether $\Delta X_{19}[9]=\Delta X_{19}[13]$ holds. Since $\Delta X_{19}[1]=\Delta X_{19}[13]$ and $\Delta Y_{19}[1]$ is known from the ciphertexts, use Lemma 1 to compute $X_{19}[1], Y_{19}[1]$ and $t k_{19}[1]$. Compute $\Delta X_{19}[15]$. Since $\Delta X_{19}[3]=\Delta X_{19}[7]=\Delta X_{19}[15]$, and $\Delta Y_{19}[3], \Delta Y_{19}[7]$ are known from the ciphertexts, compute $t k_{19}[3], t k_{19}[7]$. This step takes a time complexity of $M$ and the number of tests left for the next step is $M \cdot 2^{-c}$.

(b) Guess $t k_{19}[2]$. Using $t k_{19}[2]$, compute $W_{18}[14], \Delta W_{18}[14]$ and then compute $X_{18}[15], \Delta X_{18}[15]$. Since $\Delta Y_{18}[11]$ can be computed from $\Delta X_{19}[1,5,13]$ and $\Delta X_{18}[11]=\Delta X_{18}[15] \oplus a, Y_{18}[11]$ can be recovered by Lemma 1 , and then $t k_{19}[5]$ also. Similarly, $\Delta X_{18}[3]=\Delta X_{18}[15], \Delta Y_{18}[3], Z_{18}[3]$ can be computed from $X_{19}, \Delta X_{19}$, so $t k_{18}[3]$ can be calculated by solving the equation of the Sbox. This step takes a time complexity of $M$ and the number of tests left for the next step is $M$.

(c) With $t k_{1}[0]=t k_{19}[2], t k_{1}[6]=t k_{19}[5]$, compute $\Delta W_{2}[5], \Delta W_{2}[9]$, and check whether $\Delta W_{2}[5]=\Delta W_{2}[9]$ or not. This is a one-cell filter. Since $\Delta Y_{2}[1]=$ $\Delta W_{2}[5]$ and $\Delta X_{2}[1]$ is known from the plaintexts, $t k_{1}[2]$ can be computed by solving the equation of the Sbox. With $t k_{1}[0], t k_{1}[3]=t k_{19}[7], \Delta W_{2}[3], \Delta W_{2}[15]$ can be computed. Since $\Delta W_{2}[7]=\Delta W_{2}[11]=\Delta W_{2}[3]+\Delta W_{2}[15]$, and $\Delta X_{2}[6], \Delta X_{2}[9]$ are known from the plaintexts, $t k_{1}[2], t k_{1}[4]$ can be computed. Now $\Delta W_{2}[3,7,11,15]$ is known as well as $W_{2}[3,7,11,15]$, if the equivalent subtweakey is applied after MC. Since $t k_{2}[3]=t k_{18}[3]$ is known, $\Delta Y_{3}[7]$ can be computed and it can be checked whether $\Delta Y_{3}[7]=a$. This is a one-cell filter. Similarly, $\Delta Y_{3}[15]$ can be computed. Again, since $\Delta Y_{3}[5]=\Delta Y_{3}[8]=\Delta Y_{3}[15]$, and $\Delta X_{3}[5], \Delta X_{3}[8]$ are known from the plaintexts, $t k_{2}[1], t k_{2}[7]$ can be calculated by solving the equations of the Sbox. This step takes a time complexity of $M$ and the number of tests left for the next step is $M \cdot 2^{-2 c}$.

(d) With $t k_{19}[4]=t k_{1}[2], t k_{18}[7]=t k_{2}[7]$, compute $\Delta X_{18}[7], X_{18}[7]$ and check whether $\Delta X_{18}[7]=a$. This is a one-cell filter. This step takes a time complexity of $M \cdot 2^{-2 c}$ and the number of tests left for the next step is $M \cdot 2^{-3 c}$.

(e) Guess $t k_{2}[2], t k_{2}[4], t k_{2}[6]$ and compute $\Delta Y_{5}[1]$ and check whether $\Delta Y_{5}[1]=a$. This step takes a time complexity of $M$ and the number of tests verifying the impossible distinguisher is $M \cdot 2^{-c}$, i.e., for each pair there is $2^{-c} 14$-cell key on average that verifies the impossible distinguisher and is wrong.

The total number of tweakeys left is:

$$
T K_{\text {rem }}=2^{14 c}\left(1-2^{-15 c}\right)^{M}
$$

Brute force For the tweakeys that remain, we guess the other two tweakey cells and exhaustively search the $T K_{\text {rem }} \times 2^{2 c}$ tweakeys to find the correct tweakey.

Attack Complexities. The time complexity of the tweakey recovery phase is $M$. Following the formulas derived in Section 3.2, the data complexity of the attack is $D=2^{n+1-c} g \ln 2$ chosen plaintexts and the total time complexity is:

$$
T=D+M+T K_{r e m} \times 2^{2 c} .
$$

The memory complexity is the storage for one structure and wrong keys. For, $c=4$, we set $g=2$, then $D=2^{61.47}, M=2^{60.47}, T K_{\text {rem }} \times 2^{2 c}=2^{62}, T=2^{63.03}$ and the memory complexity is $2^{56}$. For, $c=8$, we set $g=4$, then $D=2^{122.47}, M=2^{121.47}$, $T K_{\text {rem }} \times 2^{2 c}=2^{124}, T=2^{124.60}$ and the memory complexity is $2^{112}$. 
Table 10: One of the quartets of the 15-round boomerang distinguisher of SKINNY-64-128

\begin{tabular}{|l|llll|llll|}
\hline$K_{1}$ & ed19 & f85b & $920 \mathrm{~d}$ & 6862 & 8953 & $\mathrm{f} 24 \mathrm{~b}$ & $\mathrm{fd} 90$ & $8 \mathrm{f} 60$ \\
$\Delta_{1}$ & 00e0 & 0000 & 0000 & 0000 & $00 \mathrm{f} 0$ & 0000 & 0000 & 0000 \\
$\Delta_{2}$ & 0000 & $0 \mathrm{e} 00$ & 0000 & 0000 & 0000 & $0 \mathrm{~d} 00$ & 0000 & 0000 \\
\hline$P_{1}, C_{1}$ & $8 \mathrm{ae} 9$ & $28 \mathrm{a} 6$ & 9000 & 0000 & $0 \mathrm{~b} 08$ & $912 \mathrm{a}$ & e543 & $25 \mathrm{e} 0$ \\
$P_{2}, C_{2}$ & $8 \mathrm{af9}$ & $28 \mathrm{a} 6$ & 9000 & 0000 & a4c8 & c51b bc2c & $646 \mathrm{~b}$ \\
$P_{3}, C_{3}$ & $993 \mathrm{a}$ & cad5 & $00 \mathrm{~b} 8$ & af00 & 0b08 & $912 \mathrm{a}$ & e541 & $25 \mathrm{e} 0$ \\
$P_{4}, C_{4}$ & $994 \mathrm{a}$ & cad5 & $00 \mathrm{~b} 8$ & af00 & a4c8 & c51b bc2e & $646 \mathrm{~b}$ \\
\hline
\end{tabular}

\section{B.2 SKINNY $-n-3 n$}

A 16-round distinguisher $E^{\prime}$ is placed between Round 7 to Round 21 to attack 27 rounds of SKINNY-n-3n. In the attack, $\left|k_{\text {in }} \cup k_{\text {out }}\right|=46 c, c_{\text {in }}=16 c,\left|\Delta_{\text {in }}\right|=16 c, c_{\text {out }}=15 c$, $\left|\Delta_{\text {out }}\right|=16 c$. Less than one structure is used. Suppose $2^{m}$ messages are generated under two related-tweakey respectively. Then $M=2^{2 m}, D=2^{m+1}=2 \cdot \sqrt{M}$. Suppose $\left(1-2^{-\left(c_{\text {in }}+c_{\text {out }}\right)}\right)^{M}=2^{-g}$. Then $M=2^{c_{\text {in }}+c_{\text {out }}} g \ln 2$. The time complexity is

$$
T=D+M \cdot 2^{\left|k_{\text {in }} \cup k_{\text {out }}\right|-\left(c_{\text {in }}+c_{\text {out }}\right)}+2^{3 n-g} .
$$

For $c=4$, set $g=3$, then $D=2^{15.5 c+1} \sqrt{g \ln 2}=2^{63.53}, T=2^{189}$, memory complexity is $2^{184}$. For $c=8$, set $g=6$, then $D=2^{15.5 c+1} \sqrt{g \ln 2}=2^{126.03}, T=2^{378}$, memory complexity is $2^{368}$.

Attack where tweak is used. Suppose a $w$-bit tweak is used. The tweak is loaded into the first $w$ bits of the first tweakey state $T K-1$, followed by the key material. For $0 \leq w \leq 10 c$, we have $\left|k_{\text {in }} \cup k_{\text {out }}\right|=3 n-w-2 c<2 n-w$ and the above attack still has a time complexity below $2^{3 n-w}$, so 27 -round SKINNY- $n-2 n$ is valid in such cases. For $w=10 c$, results are as follows. For $c=4$, we set $g=3$, then $D=2^{63.53}, T=2^{149}$ and the memory complexity is $2^{144}$. For $c=8$, we set $g=6$, then $D=2^{126.03}, T=2^{298}$ and the memory complexity is $2^{288}$.

\section{Boomerang Quartets}

Even though rectangle distinguishers are used to attack SKINNY, our experiment works on a boomerang distinguisher of SKINNY-64-128, since a rectangle distinguisher is valid as long as the corresponding boomerang distinguisher is valid. What's more, the probability of a rectangle distinguisher is $2^{-n} p^{2} q^{2}$, while the probability of the corresponding boomerang is $p^{2} q^{2}$ which is more practical for verification.

The experiment is implemented in standard $\mathrm{C}$ programming language. We aim at finding at least one right quartet that follow our related-tweakey boomerang distinguisher within $1 / p^{2} q^{2}$ tested quartets. For the 13 -round distinguisher, one right quartet is found among a total of $2^{12}$ tested quartets while the estimated probability of the distinguisher is $2^{-27}$. In total we found $2^{10.2}$ right quartets in a searching space of $2^{20}$ quartets. For the 14-round distinguisher, 34 right quartets are found in a searching space of $2^{40.8}$, while the estimated probability is $2^{-40}$. One of the right quartets is displayed in Table 10.

Note that our distinguisher starts from AddConstant instead of SubCells. According to our 14-round distinguisher, the plaintext difference is simply one cell difference of position 2 (we choose 1 as the actual difference value) so as to the ciphertext difference whose active cell position is 12 (we choose 2 as the actual difference value).

\section{Differential Trails}

In this section, we list the differential trails used in rectangle attacks in Section 5. Each cell (byte or nibble) of zero difference is denote by ' 0 ' and each non-zero cell is given 
in hexadecimal, ordered from left to right. $\Delta K$ is the master tweakey difference. For each round, input/output differences of the Sbox layer, as well as the the round tweakey difference are presented.

Table 11: Verification for distinguishers in Sect. 5 where distinguishers are reduce to two rounds.

\begin{tabular}{|c|c|c|}
\hline Versions & $(\hat{p} \hat{q})^{-2}$ & \#Trials on average $\dagger$ \\
\hline SKINNY-64-128 & $2^{8.42}$ & 16.08 \\
\hline SKINNY-64-192 & $2^{16.30}$ & 184.52 \\
\hline SKINNY-128-256 & $2^{15.98}$ & $3.67 \ddagger$ \\
\hline SKINNY-128-384 & $2^{19.04}$ & 29.67 \\
\hline
\end{tabular}

${ }^{\dagger}$ In each case, the average number is calculated over 10000 samples.

$\ddagger$ One reason why the numbers in this column are much smaller than expected may be that some active Sboxes can be saved as the authors of [BK09] explained. In short, it is unlikely to overestimate the probability of the distinguishers with $(\hat{p} \hat{q})^{2}$.

Table 12: Trails for SKINNY-64-128

\begin{tabular}{|c|c|c|}
\hline & 8 -round upper trail $p=2^{-12}$ & 9 -round lower trail $q=2^{-20}$ \\
\hline$\Delta K$ & 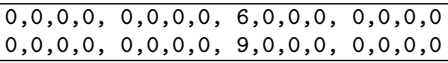 & $\begin{array}{lll}0,0, c, 0,0,0,0,0,0,0,0,0, & e, 0,0,0 \\
0,0, f, 0,0,0,0,0,0,0,0,0, & b, 0,0,0\end{array}$ \\
\hline $\mathrm{R} 1$ & $\begin{array}{llll}0,0,0,0, & 0,0,0,0,0,0,0,0,0,0,0,1 \\
0,0,0,0, & 0,0,0,0,0,0,0,0,0,0,0,8 \\
0,0,0,0, & 0,0,0,0\end{array}$ & $\begin{array}{llll}0,0,0,0, & 0,0,0,0,0,0,0,0,0,0,0,2 \\
0,0,0,0, & 0,0,0,0,0,0,0,0,0,0,0,3 \\
0,0,3,0, & 0,0,0,0\end{array}$ \\
\hline $\mathrm{R} 2$ & $\begin{array}{lll}0,0,8,0,0,0,0,0,0,0,0,0, & 0,0,0,0 \\
0,0,5,0, & 0,0,0,0,0,0,0,0,0,0,0,0 \\
0,0,5,0, & 0,0,0,0\end{array}$ & $\begin{array}{llll}0,0,0,0,0,0,3,0,0,0,0,0, & 0,0,3,0 \\
0,0,0,0, & 0,0, d, 0,0,0,0,0,0,0, c, 0 \\
0,0,0,0, & 0,0,9,0\end{array}$ \\
\hline R3 & $\begin{array}{lll}0,0,0,0,0,0,0,0,0,0,0,0, & 0,0,0,0 \\
0,0,0,0, & 0,0,0,0,0,0,0,0,0,0,0,0 \\
0,0,0,0, & 0,0,0,0\end{array}$ & $\begin{array}{lll}0, c, 0,0,0,0,0,0,0,0,0,4, & 0,0,0,0 \\
0,2,0,0, & 0,0,0,0,0,0,0,2,0,0,0,0 \\
0,0,0,0, & 2,0,0,0\end{array}$ \\
\hline $\mathrm{R} 4$ & $\begin{array}{lll}0,0,0,0,0,0,0,0, & 0,0,0,0,0,0,0,0 \\
0,0,0,0, & 0,0,0,0,0,0,0,0,0,0,0,0 \\
0,0,0,0, & 0,0,0,0\end{array}$ & $\begin{array}{llll}0,0,0,0, & 0,2,0,0,0,0,0,0, & 0,0,0,0 \\
0,0,0,0, & 0,1,0,0, & 0,0,0,0, & 0,0,0,0 \\
0,0,0,0, & 0,1,0,0\end{array}$ \\
\hline R5 & $\begin{array}{lll}0,0,0,0, & 0,0,0,0,0,0,0,0,0,0,0,0 \\
0,0,0,0, & 0,0,0,0,0,0,0,0,0,0,0,0 \\
0,0,0,0, & 0,0,0,0\end{array}$ & $\begin{array}{lll}0,0,0,0, & 0,0,0,0,0,0,0,0,0,0,0,0 \\
0,0,0,0, & 0,0,0,0,0,0,0,0,0,0,0,0 \\
0,0,0,0, & 0,0,0,0 & \end{array}$ \\
\hline R6 & $\begin{array}{lll}0,0,0,0,0,0,0,0,0,0,0,0, & 0,0,0,0 \\
0,0,0,0, & 0,0,0,0,0,0,0,0,0,0,0,0 \\
0,0,0,0, & 0,0, b, 0\end{array}$ & $\begin{array}{lll}0,0,0,0,0,0,0,0,0,0,0,0,0,0,0,0 \\
0,0,0,0,0,0,0,0,0,0,0,0,0,0,0,0 \\
0,0,0,0,0,0,0,0\end{array}$ \\
\hline R7 & 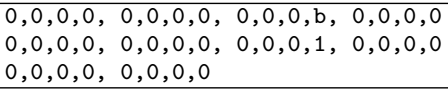 & $\begin{array}{llll}0,0,0,0,0,0,0,0,0,0,0,0, & 0,0,0,0 \\
0,0,0,0, & 0,0,0,0,0,0,0,0,0,0,0,0 \\
0,0,0,0,0,4,0,0 & & \end{array}$ \\
\hline R8 & $\begin{array}{llll}0,1,0,0, & 0,0,0,0,0,1,0,0, & 0,1,0,0 \\
0,8,0,0, & 0,0,0,0, & 0,8,0,0, & 0,8,0,0 \\
0,0,0,0, & 0, \mathrm{c}, 0,0\end{array}$ & $\begin{array}{llll}0,0,0,0, & 0,0,0,0, & 0,0,4,0, & 0,0,0,0 \\
0,0,0,0, & 0,0,0,0, & 0,0,2,0, & 0,0,0,0 \\
0,0,0,0, & 0,0,0,2\end{array}$ \\
\hline R9 & & $\begin{array}{ll}2,0,0,0,0,0,0,0,0,0,0,0,2,0,0,0 \\
6,0,0,0,0,0,0,0,0,0,0,0,5,0,0,0 \\
0,0,0, d, 0,0,0,0\end{array}$ \\
\hline
\end{tabular}


Table 13: Trails for SKINNY-128-256

\begin{tabular}{|c|c|c|}
\hline & 9-round lower trail $q=2^{-20}$ & 9 -round upper trail $p=2^{-34.42}$ \\
\hline$\Delta K$ & $\begin{array}{l}f c, 0,0,0,0,0,0,0,0,0,0,0,0,0,0,0 \\
f f, 0,0,0,0,0,0,0,0,0,0,0,0,0,0,0\end{array}$ & $\begin{array}{lll}0,0, \mathrm{cc}, 0,0,0,0,0,0,0,0,0, & \mathrm{ff}, 0,0,0 \\
0,0, f 3,0,0,0,0,0,0,0,0,0, & 9 f, 0,0,0\end{array}$ \\
\hline R1 & $\begin{array}{l}80,0,0,0,0,0,01,0,0,01,0,0,01,0,0,0 \\
03,0,0,0,0,0,20,0,0,20,0,0,20,0,0,0 \\
03,0,0,0,0,0,0,0\end{array}$ & $\begin{array}{l}0,0,0,0,0,0,0,0,0,0,0,0,0,0,0,0 a \\
0,0,0,0,0,0,0,0,0,0,0,0,0,0,0,3 f \\
0,0,3 f, 0,0,0,0,0\end{array}$ \\
\hline R2 & $\begin{array}{lll}0,0,0,0,0,0,0,0,0,0,0,0,0,0,0,20 \\
0,0,0,0,0,0,0,0,0,0,0,0,0,0,0,80 \\
0,0,0,0,0,0,0,0\end{array}$ & $\begin{array}{l}0,0,0,0,0,0,3 f, 0,0,0,0,0,0,0,3 f, 0 \\
0,0,0,0,0,0,41,0,0,0,0,0,0,0, e 3,0 \\
0,0,0,0,0,0, c 0,0\end{array}$ \\
\hline R3 & $\begin{array}{llll}0,0,80,0,0,0,0,0, & 0,0,0,0,0,0,0,0 \\
0,0,02,0, & 0,0,0,0,0,0,0,0,0,0,0,0 \\
0,0,02,0, & 0,0,0,0\end{array}$ & $\begin{array}{l}0, \mathrm{e} 3,0,0,0,0,0,0,0,0,0,81,0,0,0,0 \\
0,2 \mathrm{a}, 0,0,0,0,0,0,0,0,0,2 \mathrm{a}, 0,0,0,0 \\
0,0,0,0,0,2 \mathrm{a}, 0,0\end{array}$ \\
\hline R4 & $\begin{array}{lll}0,0,0,0,0,0,0,0,0,0,0,0, & 0,0,0,0 \\
0,0,0,0, & 0,0,0,0,0,0,0,0,0,0,0,0 \\
0,0,0,0, & 0,0,0,0\end{array}$ & $\begin{array}{lll}0,0,0,0, & 0,2 a, 0,0,0,0,0,0,0,0,0,0 \\
0,0,0,0, & 0,80,0,0,0,0,0,0,0,0,0,0 \\
0,0,0,0,0,0,80,0 & \end{array}$ \\
\hline R5 & $\begin{array}{lll}0,0,0,0, & 0,0,0,0,0,0,0,0,0,0,0,0 \\
0,0,0,0, & 0,0,0,0,0,0,0,0,0,0,0,0 \\
0,0,0,0,0,0,0,0 & & \\
\end{array}$ & $\begin{array}{ll}0,0,0,0,0,0,0,0,0,0,0,0,0,0,0,0 \\
0,0,0,0,0,0,0,0,0,0,0,0,0,0,0,0 \\
0,0,0,0,0,0,0,0\end{array}$ \\
\hline R6 & $\begin{array}{lll}0,0,0,0,0,0,0,0,0,0,0,0, & 0,0,0,0 \\
0,0,0,0,0,0,0,0,0,0,0,0,0,0,0,0 \\
0,0,0,0,0,0,0,0\end{array}$ & $\begin{array}{ll}0,0,0,0,0,0,0,0,0,0,0,0,0,0,0,0 \\
0,0,0,0,0,0,0,0,0,0,0,0,0,0,0,0 \\
0,0,0,0,0,0,0,0\end{array}$ \\
\hline R7 & $\begin{array}{lll}0,0,0,0,0,0,0,0,0,0,0,0,0,0,0,0 \\
0,0,0,0,0,0,0,0,0,0,0,0,0,0,0,0 \\
0,0,0,0,0,0,04,0\end{array}$ & $\begin{array}{lll}0,0,0,0,0,0,0,0,0,0,0,0,0,0,0,0 \\
0,0,0,0,0,0,0,0,0,0,0,0,0,0,0,0 \\
0,0,0,0,0,55,0,0\end{array}$ \\
\hline $\mathrm{R} 8$ & $\begin{array}{lll}0,0,0,0, & 0,0,0,0,0,0,0,04,0,0,0,0 \\
0,0,0,0, & 0,0,0,0,0,0,0,01,0,0,0,0 \\
0,0,0,0, & 0,0,0,0\end{array}$ & $\begin{array}{llll}0,0,0,0, & 0,0,0,0,0,0,55,0, & 0,0,0,0 \\
0,0,0,0, & 0,0,0,0,0,0,01,0, & 0,0,0,0 \\
0,0,0,0,0,0,0,01\end{array}$ \\
\hline R9 & $\begin{array}{l}0,01,0,0,0,0,0,0,0,01,0,0,0,01,0,0 \\
0,20,0,0,0,0,0,0,0,20,0,0,0,20,0,0 \\
0,0,0,0,0,0 c, 0,0\end{array}$ & $\begin{array}{ll}01,0,0,0,0,0,0,0,0,0,0,0,01,0,0,0 \\
20,0,0,0,0,0,0,0,0,0,0,0,20,0,0,0 \\
0,0,0, f f, 0,0,0,0\end{array}$ \\
\hline
\end{tabular}

Table 14: Trails for SKINNY under TK3

\begin{tabular}{|c|c|c|}
\hline & 11-round trail for SKINNY-64 $p=2^{-20}$ & 11-round trail for SKINNY-128 $q=2^{-21}$ \\
\hline$\Delta K$ & 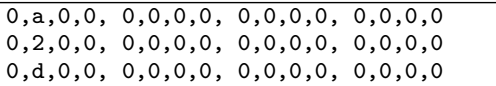 & $\begin{array}{lll}0, \text { aa }, 0,0,0,0,0,0, & 0,0,0,0,0,0,0,0 \\
0, e 6,0,0, & 0,0,0,0,0,0,0,0,0,0,0,0 \\
0, \mathrm{cf}, 0,0, & 0,0,0,0,0,0,0,0,0,0,0,0\end{array}$ \\
\hline $\mathrm{R} 1$ & $\begin{array}{ll}0,2,0,0,1,0,0,0,0,0,0,1,0,0,1,0 \\
0,5,0,0, b, 0,0,0,0,0,0, b, 0,0, b, 0 \\
0,5,0,0,0,0,0,0\end{array}$ & $\begin{array}{l}0,20,0,0,10,0,0,0,0,0,0,10,0,0,10,0 \\
0,83,0,0,40,0,0,0,0,0,0,40,0,0,40,0 \\
0,83,0,0,0,0,0,0\end{array}$ \\
\hline $\mathrm{R} 2$ & $\begin{array}{lll}0,0,0,0,0,0,0,0,0,0,0,0, & 0, \mathrm{~b}, 0,0 \\
0,0,0,0, & 0,0,0,0,0,0,0,0,0,1,0,0 \\
0,0,0,0,0,0,0,0 & \end{array}$ & $\begin{array}{ll}0,0,0,0,0,0,0,0,0,0,0,0,0,40,0,0 \\
0,0,0,0,0,0,0,0,0,0,0,0,0,04,0,0 \\
0,0,0,0,0,0,0,0\end{array}$ \\
\hline R3 & $\begin{array}{ll}1,0,0,0,0,0,0,0,0,0,0,0,0,0,0,0 \\
8,0,0,0,0,0,0,0,0,0,0,0,0,0,0,0 \\
8,0,0,0,0,0,0,0\end{array}$ & $\begin{array}{l}04,0,0,0,0,0,0,0,0,0,0,0,0,0,0,0 \\
01,0,0,0,0,0,0,0,0,0,0,0,0,0,0,0 \\
01,0,0,0,0,0,0,0\end{array}$ \\
\hline $\mathrm{R} 4$ & $\begin{array}{ll}0,0,0,0,0,0,0,0,0,0,0,0, & 0,0,0,0 \\
0,0,0,0, & 0,0,0,0,0,0,0,0,0,0,0,0 \\
0,0,0,0, & 0,0,0,0\end{array}$ & $\begin{array}{ll}0,0,0,0,0,0,0,0,0,0,0,0, & 0,0,0,0 \\
0,0,0,0,0,0,0,0,0,0,0,0, & 0,0,0,0 \\
0,0,0,0,0,0,0,0 & \end{array}$ \\
\hline R5 & $\begin{array}{ll}0,0,0,0,0,0,0,0,0,0,0,0,0,0,0,0 \\
0,0,0,0,0,0,0,0,0,0,0,0,0,0,0,0 \\
0,0,0,0,0,0,0,0\end{array}$ & $\begin{array}{ll}0,0,0,0,0,0,0,0,0,0,0,0,0,0,0,0 \\
0,0,0,0,0,0,0,0,0,0,0,0,0,0,0,0 \\
0,0,0,0,0,0,0,0\end{array}$ \\
\hline R6 & $\begin{array}{lll}0,0,0,0, & 0,0,0,0,0,0,0,0, & 0,0,0,0 \\
0,0,0,0, & 0,0,0,0,0,0,0,0, & 0,0,0,0 \\
0,0,0,0, & 0,0,0,0\end{array}$ & 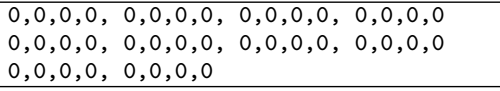 \\
\hline R7 & $\begin{array}{ll}0,0,0,0, & 0,0,0,0,0,0,0,0,0,0,0,0 \\
0,0,0,0, & 0,0,0,0,0,0,0,0,0,0,0,0 \\
0,0,0,0, & 0,0,0,0\end{array}$ & $\begin{array}{ll}0,0,0,0, & 0,0,0,0,0,0,0,0,0,0,0,0 \\
0,0,0,0, & 0,0,0,0,0,0,0,0,0,0,0,0 \\
0,0,0,0, & 0,0,0,0\end{array}$ \\
\hline R8 & $\begin{array}{ll}0,0,0,0, & 0,0,0,0,0,0,0,0,0,0,0,0 \\
0,0,0,0, & 0,0,0,0,0,0,0,0,0,0,0,0 \\
0,0,0,0, & 0,0,0,0\end{array}$ & 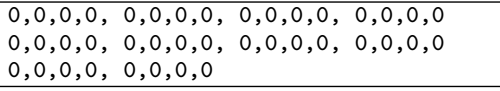 \\
\hline R9 & $\begin{array}{lll}0,0,0,0, & 0,0,0,0,0,0,0,0,0,0,0,0 \\
0,0,0,0, & 0,0,0,0,0,0,0,0,0,0,0,0 \\
0,0,0,0, & 0,0,8,0\end{array}$ & $\begin{array}{lll}0,0,0,0, & 0,0,0,0,0,0,0,0, & 0,0,0,0 \\
0,0,0,0, & 0,0,0,0,0,0,0,0,0,0,0,0 \\
0,0,0,0, & 0,0,01,0\end{array}$ \\
\hline R10 & $\begin{array}{lll}0,0,0,0, & 0,0,0,0,0,0,0,8, & 0,0,0,0 \\
0,0,0,0, & 0,0,0,0,0,0,0,4, & 0,0,0,0 \\
0,0,0,0, & 0,0,0,0\end{array}$ & $\begin{array}{lll}0,0,0,0, & 0,0,0,0,0,0,0,01,0,0,0,0 \\
0,0,0,0, & 0,0,0,0,0,0,0,20,0,0,0,0 \\
0,0,0,0,0,0,0,0 & \end{array}$ \\
\hline R11 & 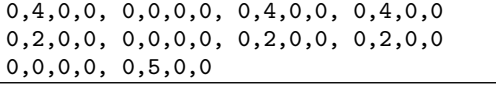 & $\begin{array}{l}0,20,0,0,0,0,0,0,0,20,0,0,0,20,0,0 \\
0,80,0,0,0,0,0,0,0,80,0,0,0,80,0,0 \\
0,0,0,0,0,83,0,0\end{array}$ \\
\hline
\end{tabular}

